\title{
Communities of practice as a curriculum design theory in an introductory physics class for engineers
}

\author{
Paul W. Irving $\odot,{ }^{1}$ Daryl McPadden $\odot,{ }^{1}$ and Marcos D. Caballero ${ }^{1,2,3}$ \\ ${ }^{1}$ Department of Physics, Michigan State University, East Lansing, Michigan 48824, USA \\ ${ }^{2}$ CREATE for STEM Institute, Michigan State University, East Lansing, Michigan 48824, USA \\ ${ }^{3}$ CCSE, Department of Physics, University of Oslo, P.O. Box 1048 Blindern, N-0316 Oslo, Norway
}

(Received 9 July 2019; accepted 9 June 2020; published 4 December 2020)

\begin{abstract}
[This paper is part of the Focused Collection on Curriculum Development: Theory into Design.] The communities of practice framework has become an essential framework for understanding identity development both in physics education research (PER) and in science, technology, engineering, and mathematics, more broadly. However, the use of communities of practice as a learning theory that informs curriculum design is significantly less prevalent within the PER community. One possible reason for this is that communities of practice as a theory originated in professional environments and it subsequently moved towards a framework that is centered around informing management practices. Some significant interpretations and negotiations need to be completed in order to apply and to design for communities of practice in the classroom environment. In this paper, we outline an introductory physics course called Projects and Practices in Physics (P-Cubed) that was designed using the communities of practice as a guiding framework. We present the curriculum decisions that focus specifically on the adaption process from professional practice to the classroom context along with the theoretical underpinnings of the curriculum design decisions that went into the development of the P-Cubed classroom.
\end{abstract}

DOI: 10.1103/PhysRevPhysEducRes.16.020143

\section{INTRODUCTION}

Within the physics education research (PER) community and the physics community, more broadly, the communities of practice (COP) framework has become widely referenced and influential when discussing an individual's experiences in classrooms, degree programs, and departments [1-4]. The framework, initially molded in the claims processing industry [5] has been adapted and applied to various types of physics and STEM contexts-from research groups to classrooms to informal learning environments $[1-4,6]$. These applications, which provide valuable insights, are often in the form of post facto investigations or justifications as to why an individual, community, or a classroom can be considered a COP or analyzed with the COP framework. From a curriculum design perspective, these studies do not provide an illustrative understanding of how the COP framework applies to the design of teachers' courses. The developers of the framework advocate reconstituting pedagogy so that it is more aligned with the situated perspective that underlines

Published by the American Physical Society under the terms of the Creative Commons Attribution 4.0 International license. Further distribution of this work must maintain attribution to the author(s) and the published article's title, journal citation, and DOI. the COP framework $[7,8]$. They argue that this can result in learning environments that infuse subject matter with more meaning to the learner than those inspired by other learning theories [5] (p. 265).

However, the COP framework has been interpreted from different perspectives. Moreover, depending on the definition of COP that one subscribes to, there might be apparent conflicts in applying the framework to the classroom context [9-11]. For example, Amin argues that COP has become a "one-size-fits-all" approach to understanding social practice no matter the context and that applying the situated practice approach to learning in every context is problematic as the socialities of knowing in action can be context dependent [9]. Similarly, Boylan argues for a reconceptualization of practice to be more flexible than the COP framing [12]. Boylan argues that "legitimate peripheral participation," which is a key concept of COP, is not "sufficient to understand either the forms of participation of participants in usual school mathematics classrooms or the reasons why the available forms of participation are as they are" [12]. These two critiques demonstrate that the COP framework can be interpreted and applied in multiple ways and that context is important to understand the application of the framework.

This is especially true when discussing the application of the COP framework to large introductory physics courses. At Michigan State University (MSU), we developed an introductory mechanics physics course called Projects and 
Practices in Physics (P-Cubed), in which the design of the classroom, messaging, activities, and assessments were all informed by the COP framework [13]. In this paper, we present the case of P-Cubed and the interpretation of the COP framework for curriculum design. In Sec. II, we present the case for designing curriculum from a COP perspective. We then briefly present the P-Cubed course design to provide context for our discussion around the interaction of the fundamental components of the COP framework with course design decisions (Sec. III). We then break the COP framework into three interrelated features that guide curriculum design: identity (Sec. V), COP theory as it relates to learning (Sec. VI), and features of and conditions for encouraging the development of a COP (Sec. VII). Sections V and VI are primarily based in the original work of Wenger and Lave and subsequent work of Wenger and iterations on that work. Section VII outlines the work since Wenger's work [5] that has focused on curriculum design. Section IX begins to focus on our context and our design by outlining assumptions we made as part of our design process. Section $\mathrm{X}$ provides a brief description of the different levels of restrictions placed on our curriculum design project due to our context. Section XI then outlines the decisions we made as part of our design process and relates those decisions to features of the COP research or framework that informed them. We close with some critiques of designing curricula using COP and with a look towards future work in Sec. XII.

\section{ADVANTAGES OF COP CURRICULUM DESIGN}

The majority of applications of the COP theory in PER have focused on identity development [1-4], particularly in exploring and understanding identity development because physics continues to have recruitment, persistence, and representation issues [14-18]. However, along with providing a space for identity development, designing a curriculum informed by the COP framework offers other advantages listed below:

(i) Space for identity development

(ii) Aligned with previous transform efforts

(iii) Creates learning environment of trust, respect, and risk taking

(iv) Facilitates a focus on practice development

The COP framework argues that being afforded the opportunities to participate in the practices of a community (physics, for example) helps to develop an identity within that community. It can provide a sense of belonging and commitment to that community [7,19-21]. Thus, one advantage to adapting the COP framework in curricular design is the ability to design for identity development explicitly. Assuming one accepts the premise that identity shapes how students participate and interact within different COP, then it is our role as curriculum designers to concern ourselves with the identities developed in our classrooms. From a design perspective, under the COP framework, learning is a transformation of one's identity. By extension, if we care about learning from the perspective of COP, then we must nurture the constant renegotiation of one's identity. From a persistence and retention perspective, designing for identity development encourages the students to reflect and to negotiate their identity concerning the subject matter in question. By encouraging the development of a COP, we can provide students a more authentic experience of what it means to do STEM and provide the supports to succeed in STEM. This focus on identity provides the opportunity for students to negotiate an identity based on what it means to do STEM. It also provides more flexibility as to what a STEM identity can be and offers the students more agency to continue in STEM as opposed to being told that they are unsuited to STEM via narrow assessments such as multiple choice exams.

From a logistics stand point, aligning with the COP framework does not involve vast changes if you are already teaching using transformed curricula. Several features or influences on the COP framework are already in line with previously developed transformed classes [22-24]. The COP framework aligns with the movement away from a centralized teacher transmitting knowledge. Instead, it argues for the creation of a learning environment in which there are multiple resources for learning [5] (p. 275). It also focuses on the power of student discussion. Student discussion is an essential tenet of the majority of transformed classrooms in physics, and a purpose for a COP is to help encourage discussion. This shift in emphasis to be more student centered and focus on student discussion, although not for the same reasons as previous transform efforts, makes the process of adapting transformed classes to include a focus on identity development a more palatable prospect.

Science can be a branch of subjects for which students hold low self-efficacy as well as instigating anxiety and low self-esteem in the students taking it $[25,26]$. Building a COP that fosters relationships of trust and respect can lead to the additional advantage of a safe and supportive environment that can enhance confidence, self-efficacy, and encourage risk taking [27-30]. Developing an environment in which risk taking is encouraged reduces the bar for every student to be engaged in the student centered discussions expected to occur.

The previous paragraph highlights the possible positive impacts of a COP that fosters a positive environment. It is essential to note that not all COPs produce positive outcomes for the participants [31] or result in the development of a positive environment. Curriculum design via COP means a focus on providing the opportunity for the development of a positive COP. In designing the COP, one focuses on offering multiple resources for learning and offering multiple opportunities for positive interactions while aligning with the learning and design goals for the course. Although a positive COP is not guaranteed, 
designed structures can be implemented in a curriculum to frequently offer and promote these opportunities.

In addition to these advantages, the focus of the COP framework on engagement in authentic practices has also been argued to be motivating and provides community members a sense of belonging to something larger than one's self $[7,24,30]$. However, the focus on engagement with scientific and engineering practices also aligns with recent calls for STEM education in America to move towards a focus on the development of practices [32]. Curriculum design through the lens of COP allows curriculum developers to take advantage of the COP structures to build learning environments that are accounting for identity development in our classrooms while also emphasizing that students learn relevant practices in engineering and physics.

\section{P-CUBED-A BRIEF CONTEXT}

P-Cubed is an introductory physics course taken primarily by science and engineering majors at Michigan State University. It is a problem-based learning (PBL) class that has elements of a flipped classroom in that students are expected to read notes or watch short lecture videos before coming to class. The class meets twice a week for two-hour periods. Each class involves solving an ill-defined PBL problem that is typically analytic in design. However, P-Cubed also has integrated computational modeling using VPython. In total, a little over a quarter of the in-class problems are computational and make use of minimally working programs (MWPs) [33]. Each week, there is a conceptually focused pre-homework designed to be completed by students before class, and there is a numerically focused post-homework that is due at the end of the week covering the topics from that week. Students take three exams during the semester and one final at the end of the semester. The final is worth $20 \%$ of their overall grade, and the other three exams, in total, are worth $30 \%$. Each of these exams splits the grade into a group portion (25\%) and an individual portion (75\%). In class, students are graded on their scientific practices and are given both formative feedback and a numerical score each week that makes up $20 \%$ of their overall grade. The remaining $30 \%$ of the grade for the class comes from the pre- and post-homework. The group portion of the exam and the in-class grading were deliberately included to communicate to students that the practice of group work is valued in this class and that it was also something that they could get better at over time. A paper that provides a more detailed description of P-Cubed from an activity and practitioner perspective is available in Irving et al. [13]. We do not want to repeat this discussion in this article. Instead, the focus of this paper is to outline how the COP framework acted as a guiding principle in our curriculum design decisions.

\section{CURRICULUM DESIGN AND COP}

Within the context of physics, there is little work from the perspective of curriculum design focused on COP. Demaree et al. lay some foundations in a paper that highlights that students need to feel that they belong to a community and need to be allowed to feel practicing physics fits with their identity [20]. However, Demaree et al. do not explore in great detail how one might design their curriculum to provide the opportunity for students to develop that feeling. The COP framework was not originally intended to apply to the classroom [7]. Instead, the initial focus of COP was on apprenticeships such as tailors, butchers, and even recovering alcoholics. In his book "COP, Learning, Meaning and Identity," Wenger extended the ideas of COP to the educational setting, which laid the groundwork for future applications to classrooms through a broad outline of its applicability to contexts of learning [5]. Wenger argues that "issues of education should be addressed first and foremost in terms of identities and modes of belonging and only secondarily in terms of skills and information" [5] (p. 263). In the COP framework, identity, meaning, and learning are not individually interpretable without the others. Thus, we will provide an outline of identity as defined by COP below (Sec. V). We then discuss learning as framed by COP and outline the dimensions of educational design as outlined by Wenger (Sec. VI). The focus then shifts to discussing more recent research that built on Wenger's work that focuses on the conditions for building and features of a COP (Sec. VII). Because of the complex interplay of learning, meaning, and identity, we will wait until we characterize the foundations of the framework before deconstructing the curriculum design decisions made for the P-Cubed course (Secs. VIII-XI).

\section{COP AND IDENTITY}

A significant part of the $\mathrm{COP}$ as a learning theory is the focus on identity as outlined in Sec. II. From Wenger's perspective, "learning transforms who we are and what we do, it is an experience of identity" [5] (p. 215). Participating in a COP is also considered not just to be a case of learning "to do" but also learning "to be" [7,12,34]. Therefore, a curriculum or learning environment designed using this framework has to include considerations for identity development. In this section, we will first provide an understanding of identity and identity development from the COP perspective. We will then highlight previous research using a COP framework in the context of science, and in particular physics, that informs our curriculum design decisions. In Sec. XI, we then outline the curriculum design decisions that are impacted by the COPs emphasis on the fundamental link between learning and identity development. 
The COP framework defines identity as a complex interplay between identity as a negotiated experience of self, a sense of membership, a learning trajectory, a nexus of multimembership, and a belonging defined globally but experienced locally [ [5], p. 264]. The COP framework creates an active link between identity and practice with the aspects of identity mentioned above having parallels in practice. This link means that elements of identity within the COP framework cannot exist without being framed through the lens of practice [5].

According to Wenger, "meaningful learning in social contexts requires both participation and reification to be in interplay" [8]. This means that each experience involves the bringing together of practice and reification in order to negotiate and renegotiate the meaning of our experience. Over time the interplay between participation and reification leads to the building of a community, where membership of that community is defined by competency in the practices, language, and norms of that community [8]. From this perspective, when a person is interacting or negotiating with a community, they are both interacting or negotiating with the community members and they can also interact or negotiate with the norms, products, and practices of that community. From this perspective, identity (as a negotiated experience) is constructed through the interaction of an individual's experiences of participation with the reification of those experiences in the form of products $[5,35]$. The identity construction is also influenced by the ways we and others reify ourselves. In the context of P-Cubed, for example, engaging in the practice of writing code through a minimally working program provides a student with experiences of participating in coding. However beyond the student's individual experience of coding, the community emphasizes particular aspects of coding, which may act to reify the student as a coder, and the student has interactions with other community members, which may also reify the student as a coder. Thus, a student can become a coder by taking on the label of a coder, but the practice that the student engages in gives meaning to what it means to be a coder in P-Cubed, and they will not be a coder until the community reifies them as such.

Identity as membership in a COP connects identity with forms of competence. A person becomes who they are by mutually engaging in the practices that constitute that community. They also take on a degree of the perspectives of the community by participating in certain enterprises. Finally, they build a set of experiences that enable an individual to negotiate with the repertoire of practice of a community [ [5], p. 245]. This is viewed as an indirect negotiation with the members of the community as the repertoire is a result of the community being built by the members through the interplay between participation and reification of members' experiences. For a student in P-Cubed, this form of identity would relate to whether the practices and norms of the P-Cubed COP feel familiar or alien. If the practices and norms feel familiar, understandable, and negotiable (that they can participate and reify in this community), then a student will build an identity of being competent within the P-Cubed community.

To understand identity as a trajectory within the COP framework, we first need to understand the interplay between the ideas of legitimate peripheral participation, trajectories, and central membership [36]. As stated previously, learning is a change in identity, and that change is akin to a trajectory where learners move from being peripheral participants in a community to being more central members $[5,7,37]$. Theoretically, for every practice that a student appropriates within a subject area, they are essentially becoming more central to a community and, in turn, develop a greater understanding of self within that community $[24,38]$. This movement from the periphery of a community to centrality is the student engaging in the legitimate peripheral practices of that community [7,39]. Whether they are becoming more central or just a little less peripheral is dependent on the practices they are appropriating, the centrality of those practices to a community, and the level of expertise they are fostering in them. For example, Irving and Sayre highlight the impact that the legitimate peripheral practice of engaging in undergraduate research had on physics majors' identities and their movement towards more central membership in the community of practicing physicists [38].

Legitimate peripheral participation refers to participation in practices in which a learner can engage that are socially warranted or legitimized by existing practitioners-the community in which the practice is situated-and are appropriate for a newcomer coming into the community to engage in Ref. [12]. The legitimacy of the practices is contingent on the community of practice being well functioning. In poorly functioning communities, the practices often lack legitimacy and just end being peripheral in nature. A well functioning $\mathrm{COP}$ is often conceived as somewhat of a dichotomy of central participants (or old timers) and peripheral participants (newcomers) within a community. An extension of this dichotomy would be that all participation that is not that of central members is legitimate peripheral participation. This conception provides a false narrative that all trajectories are inward and proves problematic when describing members who become less peripheral but are not yet central. This dichotomy has been iterated on since the initial conceptualization by Lave and Wenger [7]. There are now more types of membership classification within a community [marginal membership [5] (p. 216) or journeymen [11,40] ], but the terminology for legitimate peripheral participation has remained stagnant. There is a need for a label for participation that is postperipheral and legitimate participation seems the most appropriate. While we recognize the nuance and complexity in describing participation in a COP, for the context of 
P-Cubed as an introductory physics class, legitimate peripheral participation is appropriate for the communities that the students will be joining.

There have also been iterations in regards to possible trajectories, for example, not simply assuming an inbound trajectory for all individuals. Wenger proposed several different trajectories besides the inbound one including [5] (p. 154):

- Insider trajectories-for members who are already more central members of the community

- Peripheral trajectories - for members who seek access without the intention of becoming full members

- Inbound trajectories-newcomers who have the intention of becoming more central members

- Boundary trajectories-for members who are consistently negotiating between two or more communities

- Outbound trajectories-members who are leaving the community

Irving and Sayre found the trajectories aspect of the COP identity framework as a particularly fruitful approach to analyzing identity change within the college context [38]. For longitudinal studies, it allows for the mapping of students' places within a community over time to illustrate particular trajectories. The trajectory concept also allows for a negotiation of students' past and current experiences with future projections to understand their identity. The trajectories also illustrate the inherently temporal nature of identity as conceived by the COP framework. Within the context of P-Cubed, a student's acceptance and appropriation of practices and norms legitimized by more central members would indicate a more central trajectory. Conversely, the rejection of practices and norms would indicate a rejection and possible peripheral or outbound trajectories $[12,41,42]$.

Identity as a nexus of multimembership entails an understanding that everyone experiences membership in multiple COPs. Our behavior and the practices with which we engage differ in different COPs. However, these different behaviors, practices, and even perspectives all inform and influence our participation in other COPs [5]. Given this overlap, we must develop an identity that incorporates the different forms of participation, norms, and competencies into an accessible nexus that is called upon when participating in different communities. This nexus helps reconcile the demands of participating in multiple communities congruently and the challenge of moving from one community to another.

For learners, the work of reconciliation involved in moving from one COP to another may be the most significant challenge they face when learning in multiple unique contexts [5] (p. 159). In the college setting, individuals participate in several overlapping COPs. This fact is an essential understanding that is needed to design a curriculum informed by the COP framework. Students who are actively engaging in different COPs have opportunities to learn the knowledge, rituals, and histories valued within each community [43]. Because college students will engage in classes in different subject areas, the values, knowledge, understanding, and practices of the different communities have the potential to impact each other [5] (p. 158). This is especially true for a student who is engaged in a STEM major, where the individual STEM communities will overlap with consequence when it comes to practices. For example, Aschbacher et al. discuss the impact of the knowledge and practices learned in math affecting the practices in physics [44]. The impact can either be positive, negative, or inconsequential. Each outcome depends on the difficulty of practice transfer and the overlap in values held by the communities where a transfer could occur [45]. In the context of P-Cubed, from a curriculum design perspective, the norms, values, and practices that students are importing into the classroom must be considered as much as the norms, values, and practices they are exporting from the classroom.

Identity as a belonging defined globally but experienced locally refers to the fact that an identity formed within a $\mathrm{COP}$ is not limited in meaning to just that community. The practices engaged in on a local basis also have a meaning within the broader scheme of the more global version of that COP. Irving and Sayre's work speaks to the difference between a subject-specific identity and a professional identity, more specifically, between the physics-specific identity and the professional physicist identity [38]. For students, the local COP is the classroom where they are developing a subject-specific identity. However, the practices and identity developed in this local community have meaning in the broader community of practicing physicists. Meaning and identity between these two communities are related and both give the participant a sense of how their practices and participation fit within a broader context. In P-Cubed, students do not just engage in the practices of a physicist. Instead, there is a focus on how those practices fit within the broader context of being a physicist.

As we have described it up to this point, the COP framework frames identity primarily in terms of practice. But, Wenger also outlined alternative ways of belonging to a COP in the form of "modes of belonging" [5] (p. 173). Wenger identifies three distinct modes of belonging: engagement, imagination, and alignment. Engagement is a combination of previously discussed elements of identity: negotiation of meaning and the formation of trajectories. It is described primarily as doing things together, talking, and producing artifacts. In the P-Cubed context, this mode of belonging takes the form of mutual engagement in the solving of the PBL problems, the accumulation of shared experiences solving problems as a group, and the development of interpersonal relationships within that group. It also involves the interaction of different students' trajectories and how those interactions shape the students' identities.

Imagination is more related to the previously discussed idea of global and local COP. Imagination involves 
"constructing an image of ourselves, of our communities, and of the world, in order to orient ourselves, to reflect on our situation, and to explore possibilities" [40]. These images are essential in order to be able to orient our sense of self and our participation in the social world. In the P-Cubed context, it enables our students to produce new images of how they see themselves and their place in the world. More specifically, the students can be guided by the P-Cubed staff to extrapolate from their experiences in class to a created, potential future in which physics is an essential element of that future.

Alignment is the connection between how a member of a community is practicing and how the community might generally or historically engage in a similar practice. The connection is a two-way negotiation process and not just an adoption of external authority. From the perspective of a learner, it is how learners begin to contribute to practice. It is how they develop agency to adopt or change practice while still aligning with the traditions of the practice. In the context of P-Cubed, this could be students adapting the four quadrants planning tool, which is used to organize their work on PBL problems. Students are told to start their PBL problems by making four quadrants: facts, lacking, assumptions and approximations, and representations. However, students might adapt this tool to include a fifth quadrant or they might rename one of the quadrants so that it reflects their approach to this practice.

In summary, the COP framework characterizes identity in multiple ways and emphasizes multiple ways of belonging. In Sec. XI, we will outline how particular design choices are informed by how the COP framework defines identity. Our design decisions are also informed by previous literature in physics and science education that has used the COP framework to understand student identity development. To date, the physics education research community has used the COP framework as a lens to examine identity development through engagement with various types of experiences.

Close et al. combined the COP framework with the physics identity work developed by Hazari et al. to examine the physics identity development of learning assistants (LAs) at Texas State University [1,46,47]. The COP framework informed Danielsson's study of exploring what it means to be a woman physics student [3]. Li and Loverude's work examined students' perspectives on what it means to be a physicist or chemist [48]. More generally, Rodriguez et al. examined physics identity development in a biophysics research group in STEM [2]. Funkhouser is developing a physics identity survey for practice in laboratories informed by the COP framework [49]. Brickhouse has also used the COP framework to examine the construction of school science identities [6]. Irving and Sayre published multiple studies investigating the identity development of upper-division physics students. They looked at the impact of the learning environments and experiences the students encountered through the lens of COP [19,38,50,51].

The majority of this work focuses on establishing or measuring a change within one or multiple COP identity characteristics, and/or modes of belonging. This emphasis on change informs our curriculum design approach as it asks the question - what type of identity change are we looking to facilitate? Are we intending to impact a change in all characteristics and all modes of belonging or just some?

\section{COP AS A THEORY OF LEARNING}

Wenger describes learning as an "experience of identity" [5] (p. 151). Wenger believes that all learning is situated in a social-cultural context, and the act of learning involves the learner experiencing a change in their identity by engaging in the social practices of a community $[5,7,34]$. "Situated learning," the terminology used by Lave and Wenger [7,52] to describe COP as a learning theory, is a movement away from the perspective that learning is just the acquisition of knowledge [12,53-55]. Situated learning is the progeny of anthropologists and cognitive scientists due to a convergence in each field on the importance of context to understanding how individuals learn $[24,52]$. It is this emphasis on context and the influences of these two fields of research that place participation in practices that are socially situated at the core of what it means to learn in a COP. From this perspective, learning is a social and collective activity. Thus, the understanding and the knowledge that is developed by an individual is really a shared understanding and knowledge that is learned through participation within a group and the adoption of shared practices [56,57].

There are a lot of interlocking parts to the definition of learning from the COP perspective. To summarize, learning is a social and collective activity. An individual develops knowledge and understanding by participating in and adopting the shared practices of a community. The knowledge and the understanding developed is a shared knowledge and understanding because it is developed in conjunction with and communicated to the community as the learning occurs. The learning results in a meaningful change that can manifest in the following forms: the change of the learner's relationship to practice, the change of the learner's relationship to other people engaged in that practice, and the change in the learner's identity due to participation in the practice.

In the P-Cubed environment, students solve complex problems in groups while being guided in their practice by a more central member of the community in which they are participating (typically an LA). They engage in many practices in collaboration with their group mates during each problem session. If the focus of the learning was to develop an approach to solving energy problems, then coordination of the perspectives and actions of the group to 
develop an approach must occur. Then the individual must negotiate meaning in the approach that is collectively developed by their group. Because this negotiation is an interaction that is occurring simultaneously in conjunction with the community-negotiating the approach means negotiating with the community. They must both reify and contextualize the meaning of the approach in order to complete the negotiation process and effect a meaningful change. The reification could come in the form of a stepby-step plan that could be broadly applied. The contextualization could occur in the form of identifying where the ability to solve energy problems fits within physics more broadly or that the approach developed could be applied in more than just energy problems. An identity change could occur from the knowledge they have obtained about energy and how it fits with their understanding developed in their engineering COP.

Even though the main focus of learning in $\mathrm{COP}$ is in the change of an individual's identity, the acquisition of knowledge still occurs. Constructivism is an influence on our understanding of knowledge obtainment in COPthe focus of learning is moved away from the teacher and centered upon the learner [22-24].

\section{A. Dimensions of educational design}

A caveat for curriculum design is that the emphasis on the context within the COP framework can be problematic when transferring the framework to the school setting. In school, the relationship between context and practice is frequently stripped away so that the learner's relationship to the practice is less meaningful [5] (p. 268). Learning as participation in practice changes the rules for designing curriculum. It centers curriculum design around providing students the opportunity to participate in collaborations, developing interactions based on shared goals, and building a COP $[58,59]$. To scaffold the design of learning environments (context) that provide the opportunity for meaningful transformation, Wenger outlined four dimensions of educational design and interpreted the three modes of belonging (see Sec. V) for learning environments. Below, we outline these dimensions with examples of how they influenced design in the P-Cubed context. In the next section, we discuss how the modes are part of many conditions for building a COP.

According to Wenger, operationalizing COP as a curriculum design framework involves the work of engaging with the four dimensions of educational design where each dimension represents a duality [5] (p. 264). Designing a curriculum with these dualities in mind is not meant to represent a choice between two opposing poles. Instead, the dualities inform us that we need to combine these poles productively in order to address the tension between them. The four dimensions are participation and reification; the designed and the emergent; the local and the global; and identification and negotiability.

\section{Participation and reification}

Wenger argues that education has traditionally leaned towards the reification pole. This reification occurs in the forms of a prescribed curriculum or textbooks. Guided by the principle of COP, designers should question when they should reify and when they should instead rely on participation. Inevitably, education involves the reification of material or subject matter; however, we must find a balance with the design of a learning environment or curriculum such that it provides forms of participation that invite entry into a practice and let that practice be a substantial part of the curriculum. Participation in practice provides the opportunity to negotiate meaning in the students' learning that encourages the students to invest a part of themselves in the process in the form of identity transformation.

To strike a balance between reification and participation in the P-Cubed learning environment, the class was designed to focus class time around participation in practices explicitly. The PBL design means that there is no passive listening to previous subject matter reifications in class, and instead, the students are engaged continuously in practices such as problem solving, group work, planning, and decision making. Reification still occurs, often in the form of students' solutions to the complex problems, but this reification is often student centered. Their answers are not dependent on some pedagogical authority. Instead, the answers are negotiated by students based on their interpretation of the problem and the assumptions that they have made.

\section{The designed and the emergent}

The duality of the designed and the emergent positions the relationship between teaching and learning as something beyond cause and effect. Learning is not a reaction to teaching. Instead, it is a response to the pedagogical intentions of the learning environment and is highly dependent on the context. The goal of instruction is not to impart knowledge and understanding to the masses. It is to create a context in which learning can take place. From the COP perspective, teaching, learning, and practice are all linked via resources and negotiation. Resources typically refer to the teachers and instructional materials that they provide, but resources are also the product of the interaction between teaching and learning. They are scaffolds that are produced to facilitate interaction between the planned and the emergent. Design from this perspective is striking a balance between these dualities where there is an emphasis on honoring the emergent nature of learning while also acknowledging that every context needs some element of scaffolding in order for learning and teaching to inform each other.

The P-Cubed learning environment is influenced heavily by the idea of creating a context for learning to occur. The primary example of this influence is the decision to focus all in-class activities on group interactions while also 
making use of problem-based learning and minimally working programs (for computational modeling activities). We designed the PBL and MWP problems as a scaffold from which students' ideas and understanding can emerge and be interacted with in order to develop their own unique solution to the problem. The ability of the students to decide their learning path is due to emphasis on creating PBL and MWP problems that are underconstrained or design based. Students then make decisions and assumptions that constrain the problem. These constraints will bring about productive conceptual discussions within the group about why the decision they made is appropriate or why an assumption is useful or sensible. This process then leads to the development of an understanding of the underlying physics principles. We explicitly communicate to the students via multiple media that a final answer is unimportant and that we care about the journey to the answer. Learning goals are negotiable depending on the path students choose to take. The conceptual learning goals of the class broadly align with three conservation principles: conservation of momentum, conservation of energy and conservation of angular momentum. As long as the teaching staff believe a groups learning goals for a day will align with one of these principles or result in the opportunity to develop in a scientific practice then they will be encouraged to choose it as a learning goal. The teaching team is aware that the next activity will typically offer an opportunity to encourage any meaningful conversations that did not occur in the previous one.

\section{Local and global}

If the designed and emergent duality emphasized the importance of context, then the local and global duality emphasizes the importance of ensuring that the learning that occurs transcends that context. Learning in COP is a result of a transformative experience that results in some level of identity renegotiation. Thus, what students are learning needs to have a broader scope of relevance in order to inspire a renegotiation. The focus should be on providing students with transformative experiences that involve engaging in new forms of membership, multi-membership in different communities, or the opportunity to negotiate meaning that holds some significance beyond the context in which the learning is occurring. This duality also emphasizes a focus on metacognitively developing students to the point where they can learn no matter the context. In essence, students would develop the skills and practices of a lifelong learner.

In the P-Cubed context, we attempt to connect the local to the global by focusing our course-level learning and design goals on developing expertise in the overlapping practices of both science and engineering. For example, working successfully in a group is inherent to success in both engineering and scientific disciplines. We communicate this truth via evidence from both scientific articles and personal reflections at the beginning of the semester in our introduction day. We then reinforce this idea via various forms of messaging. Our definition of messaging is everything communicated directly to students about our motivations for activities they work on inside and outside the classroom. We then provide the opportunity for students to engage in the practice of group work consistently throughout the course. The design incorporates essential aspects of group work such as decision making, developing group dynamics, and planning in groups. Students then receive individual feedback that guides them in developing competency in these aspects of group work. However, groupbased learning in the P-Cubed context intends to apply to future or concurrent contexts both at the undergraduate, postgraduate and professional levels resulting in hopefully an authentic connection between the local and global.

\section{Identification and negotiability}

The final duality is a focus on identification and negotiability, which refers to a learning context offering "meaningful forms of membership and empowering forms of ownership and meaning" [5] (p. 269). Wenger refers to the importance of a curriculum designer attending to identity and related issues when building a learning environment within an institution. Designers should consider sources of identification offered both in a learning environment and within the institution. They should further consider how those identities compete with each other. Designing under these considerations should include projecting forward to how participation might evolve as participants continue on their trajectories. It also means considering how participation in multiple communities will impact identity within the designed learning environment and the cross-cutting communities with that environment. If new trajectories of participation are not part of the design process, then communities will remain stagnant. The result could be a learning environment that only invites participants who already identify with the community to develop within that community, which can result in the alienation from either the designed learning environment or, correspondingly, the institution in which the learning environment exists. From a negotiability perspective, opportunities for negotiation with both the community artifacts and people offer the ability to shape the enterprise and practice of a community. The ability to negotiate in these fundamental elements of a community translates to a sense of ownership of the community.

In the P-Cubed context, opportunities for negotiation are challenging to include due to institutional restrictions of the physics department. Furthermore, the course's introductory nature competes with being able to provide students with the ability to shape the enterprise and practice of the community. Our attempt to include the opportunity to shape the enterprise of the community is in the flexibility designed into the P-Cubed problems, which often ask 
the students to interpret the goal of the problem for themselves. This flexibility perpetuates through to the group exams where students are again asked to define the problem. We also include the ability to shape practice through how students are able to reshape the scaffolds that we put in place to guide the development of students' scientific practices. For example, the four quadrants' planning tool is typically adapted to include the elements that students find useful over time and to eject aspects that they find redundant. In regards to identity, we are attempting to build a connection between physics and engineering identity to make apparent the overlapping nature of these communities. We identify cross-cutting practices in an attempt to form a connection of relevance between identities so that the identities do not necessarily compete but complement each other. We still keep in mind that these practices have to be negotiated when transferred from one context to another.

In the following section, we describe how these dimensions have been iterated on by researchers and curriculum designers or interpreted for building an understanding of the conditions needed for a COP to develop.

\section{FEATURES OF AND CONDITIONS FOR BUILDING A COP}

Scholars disagree about whether it is possible to design a COP [10,60-62]. However, there is agreement that COPs can be cultivated and supported in their development $[56,63,64]$. Thus, it is possible to design a learning environment where there is an opportunity for a COP to develop. In order to sprinkle the seeds of growth, we must understand the fundamental features of a COP. The recognizable features of an existing $\mathrm{COP}$ and how those features become established has evolved since the first presentation of the framework by Lave and Wenger [7]. Wenger created the groundwork for the features and conditions for a COP to exist by aligning curriculum design with identity through the modes of belonging introduced in Sec. V. Wenger argues that designing with the modes of belonging in mind transforms teaching to being concerned with the effects of identity formation as opposed to just the delivery of a curriculum. From this perspective, learning environments are to be designed to connect to the world in meaningful ways. According to Wenger, to do so, the following conditions need to be met:

- Students need places of engagement-educational engagement

- Students need materials and experiences with which to build an image of the world and themselves-educational imagination

- Students need ways of having an effect on the world and making their actions matter-educational alignment

However, subsequent to the presentation of these conditions, there has been a large body of work that has iterated, contextualized, and added to these conditions in order to understand how to encourage COP development [22,65-67]. The following outlined conditions appear in literature the most frequently but have their origins in both the academic and corporate instantiations of the framework. In some cases, we have grouped conditions based on similarities in theme and linked back to Wenger's original outline. The features and conditions outlined below are central for a productive COP to prosper. We present the features and conditions in no particular order as we are not aware of any research that has attempted to establish the prominence of one feature over another. What follows are a cultivation of conditions for a COP with commentary on the relationship of each to the development of a curriculum.

\section{A. Shared area of interest or a sense of joint enterprise}

Across all descriptions of COP, a fundamental feature of a community is that they share an area of interest or a sense of joint enterprise $[5,9,22,65,66,68-70]$. To describe in a more concrete manner, an area of interest in the COP framework is a common focus for the community that can be as simple as a concern that needs to be addressed or as complex as a set of problems that need be solved. More broadly, it might be a shared passion for a particular topic. This shared area of interest informs the group's identity and, by consequence, an individual's identity when they are participating in this community. An example, from our previous research, is instructors coming together as members of a community (i.e., the Partnership for the Integration of Computation in Undergraduate Physics, PICUP) with the shared interest to integrate computation into the undergraduate curriculum [67]. The related feature of joint enterprise, interprets this feature as being more focused on community members'understanding of how they are connected [58]. The joint enterprise of the community is not stagnant, and instead, community members renegotiate the enterprise during the life of the community [71]. The joint enterprise is akin to the shared interest perspective. The connection between community members concerning a joint enterprise is often a shared interest. However, from the joint enterprise perspective, the connection can also be a shared understanding or history that connects the members. We grouped these two features together because of the similarity in how they describe a COP. These features do differ due to a chicken-and-egg like distinction. From one perspective, the community comes together because of shared interest. But from the other perspective, the community interacts in order to develop a shared enterprise. The result of either perspective is that the community has a shared desire that informs the community's interactions.

From a curriculum design perspective, this feeds into decisions about the type of learning paradigm you want, the structure of activities, and the messaging communicated by the teaching staff. The central members of the community need to establish the possible joint enterprises for the 
potential community to engage in. This establishment can come in the form of activity design or communication around learning goals for those activities. On a broader scale, establishing possible joint enterprises can come from the communication about the design goals for the entire class. By design goals here, we mean goals that are intended but that are not going to be explicitly assessed. Learning goals and design goals operate on different scales and could result in the formation of different COPs within the same context. In the P-Cubed context, this could be a COP at the level of the whole class or the level of an individual group.

There is no direct connection between these features and Wenger's modes of belonging. But, we would argue that a shared area of interest or joint enterprise aligns with aspects of educational imagination and educational alignment. With respect to educational alignment, the shared interest or goal of a community is going to effect the type of impact one can have on the world and, thus, influence how one's actions within a community matter. Considering educational imagination, it is having a sense of joint enterprise that informs the types of experiences that the students are going to have access to in order to build an image of the world.

To contextualize this condition in the P-Cubed classroom, we attempt to communicate that the joint enterprise is the development of conceptual understanding and proficiency in scientific and engineering practices. Our attempts to communicate this are through learning goals, assessment practices, and messaging (i.e., group interactions, the syllabus, and an orientation presentation at the beginning of the semester). However, there may be a difference between the joint enterprise we communicate and what the students negotiate to be the joint enterprise. For example, at a group level COP, one could imagine a joint enterprise negotiated that is "finishing the PBL problem as quickly as possible." This joint enterprise would be based on what each group member's shared interest is within the group level COP and how that interacts with the class level COP.

\section{B. Mutual engagement}

Beyond the shared area of interest, both Wenger and subsequent research has centered COPs around mutual engagement $[5,8,36]$. At its most basic, mutual engagement is often referred to as the opportunities for shared participation in the activities in which the community engages. Research describes mutual engagement as the binding agent between community members that develops the community into a social entity [69]. Descriptions of mutual engagement often center on relationships between community members and the norms, expectations, and reifications that they have made or built over time through engaging in shared practices [56,57]. Mutual engagement is not just the shared participation that occurs within the community. It is also a description of the culture within which both the participation is occurring and how community members develop that culture through their ongoing interactions. A cultural component emphasized within the COP literature for mutual engagement is one of trust $[66,69,72,73]$. Trust is emphasized in previous literature because a COP should offer individuals the ability to explore new ideas or new approaches to practice without the potential to feel embarrassed [ [5], p. 214]. Trust allows for the emergence of radical or out-of-the-box ideas [69]. Previous research has demonstrated that trust is fundamental to promoting higher levels of openness in groups, which can increase the efficiency of knowledge transfer within a group [74]. Another cultural component concerning mutual engagement is communication. Communication is connected to trust as more frequent and inventive communication can occur when trust is held between group members. But, communication is also discussed from the viewpoint of information sharing and the speed or accessibility of that sharing. There is a particular emphasis on how information is being used to make decisions within the community $[5,9,22]$.

From a curriculum development perspective, mutual engagement means that students in a community should be engaged in shared participation within a communication space that promotes a culture of coresponsibility and trust. It also means, referring back to the designed and emergent dimension discussed above, that the mutual engagement (participation) that students engage in must give meaning to the subject matter without over reifying the subject matter. Considering mutual engagement in these ways will support students in understanding the community of which they are members and how to succeed within that community. Connecting back to Wenger's modes of belonging - the guidelines indicate the need for opportunities for mutual engagement and including members in shared practice. Mutual engagement and shared practice are essential for a COP to exist and are categorized by Wenger as falling under the educational engagement mode of belonging [5] (p. 271).

In the P-Cubed context, on a fundamental level, the class is entirely group orientated, which facilitates mutual engagement in a shared communication space. The students are asked to reify the subject matter through their group work by negotiating the critical elements of their work that they would like to reify for themselves, if any. This lack of directed reification translates to students deciding how much of their learning needs to be reified in the form of notes, photos, or reports. We attempt to encourage a culture of coresponsibility by making sure to communicate that we are not grading the students on a curve. Instead, students' final grades are determined by a fixed scale. This encouragement attempts to reduce the emphasis on competition. The group-based formative feedback rubric also emphasizes the need for collaboration and 
personal responsibility to the group's learning and understanding.

\section{Shared repertoire}

Within the COP framework, the conditions of joint enterprise, mutual engagement, and shared repertoire are highlighted by Wenger as the only conditions necessary for a COP to exist [5]. The meanings of these terms are often interrelated, with trust specifically discussed within both shared repertoire and mutual engagement. For curriculum design, trust is an essential part of the design, we chose to place it in the mutual engagement description as it seemed the most appropriate place. The core of our discussion on shared repertoire will focus on enculturation, cultural artifacts, and language. Barab has argued that for a sustainable COP to exist (one that persists over time always promoting new central members) then there must be a significant culture and history into which to become enculturated [24]. The majority of the culture is preexisting and inherited by new members to the community in the form of previously negotiated goals, meanings, and practices. But, there is also a negotiation process that allows for the evolution of the culture of the community through newcomer participation and negotiation [29,75]. Language is an example of a shared repertoire that is fundamental to practice within a community. Obtaining access to the language of the community is fundamental to developing an identity related to the community and is a sign of movement from the periphery of the community to more central membership. In essence, a community needs to have its language in the form of jargon, shortcuts, representation conventions, and terminology, as it provides a stabilizing element to the community [22]. In the same vein, other cultural artifacts such as local lore, shared stories, and inside jokes also represent opportunities for enculturation $[5,12]$. Communities also construct artifacts via reification $[5,76]$, which, as described above, is the process of developing physical and conceptual artifacts that reflect the shared experience of the community. The artifacts then become central to participation within the community.

From a curriculum design perspective, there must be a culture into which one is enculturated; however, there are decisions around which communities (and thus cultures) we are attempting to align with our design. From a pedagogical perspective, it is also vital that teaching staff are aware of the culture of the communities for which they are acting as central members. There are also implications for design decisions around cultural artifacts and putting in place artifacts that can be culturally appropriated and adapted by new community members.

Referencing back to Wenger's conditions of a COP, "shared repertoire" is a spin on the educational imagination condition. We would argue that the culture that must exist for students to enculturate into is made of the same materials and experiences that students will use to build an image of themselves and the world.

In P-Cubed, an example of a cultural item that is both pre-existing but also negotiated over time is the role of assumptions. Starting with the first PBL problem, students have to make assumptions in order to narrow the focus of the problem and produce their particular solution. However, what constitutes an appropriate assumption is something that the students negotiate over time by engaging in discussions with each other and teaching staff. By producing reifications of what it means to make assumptions in the form of group exam solutions or as part of their four quadrants white board, students further negotiate the role of assumptions.

\section{Learning from near peer and central members}

Outside of joint enterprise, mutual engagement, and shared repertoire, the presence of opportunities to learn from more central members of a community is the most referenced condition for a COP to exist $[7,9,24,66,77]$. It is essential to colocate newcomers with more experienced members of the community in order for them to benefit from the different levels of expertise that are present in the COP. This practice provides old timers with the opportunity to coach and model thinking skills for newcomers. The mixed levels of expertise are a fundamental social structure of a COP, which facilitates the development of newcomers' identities and their movement from peripheral to full membership. The structure of this apprenticeship type model is essential to a COP because it provides the community with the ability to reproduce $[24,30,78]$. New members engage in the practices of old members and become more central until they then replace the older members of the community and, in turn, act as guides into central membership. This process also ensures that the rituals and cultural intricacies of the community remain a part of the community [24,56,79].

From a curriculum design perspective, it is essential to consider the makeup and role of the teaching staff within the classroom COP. It is also essential to take into consideration the type of members present in the COP when designing the learning groups.

There is no direct comparison to the conditions Wenger outlined that are related to his modes of belonging. However, Wenger does categorize the role of old-timers as an educational resource. He also cautions a need to ensure a community in which educational experience and dynamism of youth are balanced when engaged in the same practices. [5] We will not provide a specific contextual example of this condition in P-Cubed here as a whole section emphasizes addressing this condition as part of our design in Sec. XI.

\section{E. Feeling part of something larger}

Both Barab and Wenger outline the importance of community members believing that membership in the 
community results in them being part of something more substantial [5,24]. From Wenger's perspective, being part of something more substantial returns to both the local and global duality (discussed in Sec. VI), and the imagination and alignment conditions (discussed in Sec. V). The literature since Wenger's work also related the feeling of being a part of something larger to the individual becoming a member of the community and the community being nested or interrelated with other communities $[9,24,30]$. From the individual perspective, the literature argues that a person's motivation for wanting to join a COP is that by joining the community and engaging in the practice of the $\mathrm{COP}$, they become enmeshed with that community. A COP addresses becoming a part of something more substantial by providing the ability to invest in this new community and its goals with people who have shared values. From a nested community perspective, the COP needs to have a relationship with the larger world. This need connects back to Wenger's educational alignment condition and the need for an individual to have an impact more broadly than just the local community. How a COP functions in the context of society gives the community and individuals in the community an understanding of how their particular goals and identity fit globally.

From a curriculum design perspective, the "part of something larger" condition influences decisions about messaging from the teaching staff as well as the authenticity of the activities. Perceiving class-based activities as belonging within practices of a broader community will allow for connections between communities of different sizes. Ensuring consistent messaging about how the practice in the classroom relates to professional practice can be folded into design features such as feedback and class level messaging (discussed further in Sec. XI).

A P-Cubed example of a connection to a broader context is the emphasis placed on the practice of planning. On the orientation day for class, we highlight the importance of the practice of planning within both the engineering and science disciplines. Research literature, program documents, popular magazines, and shared experiences from teaching staff are all used to emphasize the importance of planning within the work of science and engineering at every level. We then emphasize the importance of planning again within the local context when students are working problems. This is done through the teaching staff's facilitation of the groups as well as their feedback delivered to students each week. We attempt this link between global and local contextualization of practices with all the scientific and engineering practices that are learning goals for the class.

\section{F. Ensuring COPs are collaborative and involve engagement with authentic activities}

Based on the previous assertions in the COP learning theory (Sec. VI) that both situated learning and constructivist theories inform COP [22-24], it seems relatively straight forward to assert that a COP should be both collaborative and involve authentic activities. The COP literature argues that knowledge that is held and developed in a collaborative process is more significant than knowledge developed and held by an individual $[22,80]$. Because mutual engagement is an expected feature of a COP, this should mean that the community is inevitably collaborative [81]. Previous COP literature has also asserted that practices and activities within a COP should be purposeful, allow for the building of meaningful discussion, and be assessed authentically [82]. Assessed authentically here refers to being explicit about the connection between central and personal practice.

The example of planning that was illustrated in the last section offers an additional connection here. The students who come into P-Cubed often have a conception of planning that can be as limited as believing that planning involves only identifying your final goal. However, the central practice of planning will provide an extensive list of steps to be taken and evaluation points that are built into the solution process. The assessment for planning is designed to scaffold the movement of our students to becoming experienced planners via explicit feedback on planning through formative feedback based on in-class work, inclass guidance, and feedback on group exam solutions. This focus on authenticity and authentic assessment facilitates newcomers' trajectories towards central membership through the development of perceptions of what central membership means [38]. The need for authenticity informs curriculum development decisions around assessment and activity design. It also informs the design of the individual groups in P-Cubed. The activities must be authentic to both the COP in the classroom and any relevant overlapping communities.

\section{CURRICULUM DESIGN WITH COP}

The literature and theory outlined up to this point indicates the complexity of the COP framework, especially when attempting to apply it to the classroom setting. We deliberately outlined COP from the perspectives of identity, learning theory, and conditions for building a COP in order to reference what is informing our curriculum design decisions. However, when designing our curriculum, we did not try to adapt our design to be a full comprehensive instantiation of the COP framework in our classroom. Instead, we based our curriculum design on several assumptions, the restrictions that we perceived our context to be enforcing, and the curriculum design decisions that we made. The following sections outline these three different aspects of our design.

\section{ASSUMPTIONS MADE TO APPLY COP TO OUR CONTEXT}

Applying the COP framework to the college classroom setting involves several interpretations of critical features of 
the framework. The COP framework was initially developed in industry, and we need to modify several of the features of the framework in order to translate them to the educational context. Below we discuss interpretations, assumptions, and modifications of the framework that we made before applying COP in our curriculum development project.

\section{A. Individual Classes can be a COP}

It is essential to highlight that no individual participates in only one COP. Instead, individuals participate in several overlapping COPs and, occasionally mutually exclusive ones. More accurately, individuals can participate in COPs that are tangential to the community we are attempting to develop $[7,37]$. This assertion leads into the first significant interpretation of the COP framework. In the college context, COPs can exist on the individual class level and therefore class-based COPs can be overlapping $[50,83,84]$. This interpretation is shared at least in part by some of the community researching COPs as well as some of the first author's previous work, which demonstrated how an advanced laboratory class developed and existed as a COP [50,83-86]. Building from the classroom as a COP interpretation, we will assume within our context that other, more micro-COPs can develop. These could manifest in the form of a COP of each group of four students or potentially a COP encompassing a staff member and the groups they are working with exclusively.

This suggests that when applying COP to our classroom space, there are multiple overlapping communities to be considered from the group-level COPs to the class-wide COP. These COPs can exist at different scales: the microscale of the group, the more mesoscale of the classroom itself, and the macroscale of the COP of the discipline. From a curriculum design perspective, we are interested in ensuring that the conditions for building a COP are present within the micro- and mesoscales, so that participants in those communities can build a COP. As we will argue below, the macro-community is truly a pair of macrocommunities when considering curriculum design for our context. Both of these communities refer to the COP within the possible future fields for our student body: the physics COP [38] or the engineering COP. From this perspective, one views the classroom as an opportunity to engage in the practices of a broader community. The macro-, micro-, and mesocommunities are not mutually exclusive, as it might often make sense for a sub-COP to be contained within a broader COP [38]. For example, students engaging in the practices of an advanced physics laboratory COP (a mesoscale classroom COP) is likely also engaging in the broader community of practicing physicists (a macroscale $\mathrm{COP}$ ) due to the overlapping nature of the practices and shared goals of the communities. However, it is also true that every classroom does not have to be a COP in order for the COP framework to still inform classroom curriculum design. The choice of perspective on what is or is not a COP would be dependent on the intended design and learning goals of the curriculum project.

\section{B. Overlapping COP}

The overlapping nature of COP within the college setting also has repercussions for identity development with different identities being harbored and developed within separate communities. Irving and Sayre's work speaks to the difference between a subject-specific identity and a professional identity, more specifically, between the physics-specific identity and the professional physicist identity [38]. Irving and Sayre have defined a subjectspecific identity previously as one formed through the combined membership of all the physics undergraduate classroom communities that a student is taking. A subjectspecific identity does not refer to full central membership in the community of practicing physicists. Instead, it is a stop on their trajectory to becoming central members of the community of practicing physicists. Thus, the identities are related but different due to COPs that are influencing their identity change. It is important to remember that there are multiple COPs contributing to the development of a subject-specific identity. Furthermore, the COP of undergraduate physics students is more closely aligned to developing a subject-specific identity than to developing a professional physicist identity. Irving and Sayre argued that a student's subject-specific identity in physics is part of their trajectory to becoming a central member of the community of practicing physicists. If students are taking multiple majors, they are building multiple subject-specific identities by engaging in the community practices of their subject majors. The interrelationship and outcomes of having many subject-specific identities have not been investigated. However, when a student is only engaged in the practices of communities that develop a subjectspecific identity in physics, there is a limit to the amount of identity development that can be done in the community of professional physicists. For example, engaging in problem solving that is focused on developing simultaneous equations and doing well on a presentation are legitimate practices of the COP of undergraduate physics students and also legitimate practices of the community of practicing physicists. However, conducting and presenting research, writing grant proposals, and contributing to a research group are more likely to be more central practices of the community of practicing physicists.

\section{Instructor as the central member-old timer}

Applying the COP framework to our setting requires us to make assumptions about who are the central members of the community and, therefore, who provides guidance into the community. From a design perspective, we are assuming different levels of centrality for each of the different types of teaching staff within the P-Cubed context. This 
assumption means that there is likely variability in the possible trajectories that the different teaching staff can offer to their students. However, this is variability in the local to global connection and not within the COP intended to develop within the P-Cubed classroom. From the potential P-Cubed COP perspective, the instructor, grad students, and LAs still act as more central participants of the communities that develop in the P-Cubed classroom. They are all guiding peripheral participants on a trajectory to the more central membership within the classroom community $[87,88]$.

\section{Crucible courses and accelerated trajectories}

Just like aircraft can have different trajectories due to different velocities, so too can students have different membership trajectories due to different opportunities. In previous work, Irving and Sayre have argued that some classrooms can provide more significant opportunities to engage in practices that are more central to a community than other classes and courses [50]. By extension, engaging in more central practices means also receiving guidance in those practices. They called these classes, "crucible courses," and described them within the context of physics as "the first courses in which students work on difficult physics problems surrounded primarily by other physics students, where their professors treat them as junior physicists, and they take on identities as part of the broader community of physics undergrads." Irving and Sayre also argued that these courses involved a substantial change in accountable disciplinary knowledge, where what it means to do physics or what counts as doing physics substantially changes along with students' physics identities $[50,89,90]$. For example, being good at physics can change from doing well on physics exams to being able to carry out sophisticated experiments and reflect on the data collected to writing a peer-reviewed journal article.

In regards to curriculum design, these courses provide students with opportunities to engage in more central practices in a social space where they can be welcomed into the central community by peers and members of a more central standing. We refer to this engagement in more central practices and the acceptance by more central members through an experience that would not typically offer this form of access as an "accelerated trajectory." However, opportunities do not have to be taken up, and central members do not just bestow legitimacy by providing acceptance and guidance. The students themselves have to see the practices that they are engaging in as legitimate to them [38]. Therefore, a classroom can provide the opportunity for an accelerated trajectory but not force one, which can often result in different students having varied trajectories as a result of taking a class.

Irving and Sayre previously made the argument that designing an introductory physics class to provide an accelerated trajectory would be inappropriate because of the probable massive shifts that students are experiencing in their first year of college [50]. Students would have to contend with not just the adjustment to the norms of college but also, if it is a transformed learning environment, grapple with a movement away from how accountable disciplinary knowledge has changed from their previous high school context. In the P-Cubed context, the focus moves away from traditional approaches to understanding concepts, developing aptitude with practices, and constructing understanding socially.

It is also true that an introductory class would be a very peripheral point to start your trajectory into the subjectspecific community. In the introductory context, students are either on the path to entering or having a flirtation with the subject, and so it would be unreasonable to expect large accelerations into centrality. That does not mean it would be inappropriate to design an introductory physics class with the COP framework. Instead, it just means being judicious about how much of a central trajectory the design should offer in these formative classes so that these initial classes are not too overwhelming. For example, the practice of creating a model, especially in regards to the assumptions that go into that model, is an essential practice of doing physics. The version of model creation and assumption making that happens in P-Cubed is a different level of authenticity than what this looks like in experimental physics. By engaging with this practice at the introductory level and providing an understanding of the importance of it to "doing physics," we are providing the opportunity to engage in this practice in a meaningful and still manageable way.

\section{RESTRICTIONS BASED ON OUR CONTEXT}

No curriculum design project exists in a bubble. Department, college, university and national norms and requirements all influenced the decisions we made during this design project. A further restriction is the designers own experiences of designing and teaching similar types of classes. No matter the intentions sometimes personal experience outweighs prescription to learning theory.

The departmental constraints placed on us included concerns around content coverage, the norms around the number of exams expected for this class, and the use of homework grading systems. These norms came into conflict with the COP framework when it came to our intention to focus on practice. From our personal experience, a lot of transformed curricula result in alternative learning or design goals being in direct conflict with the amount of content being covered (for example, focusing on group work). Our focus on practice development did result on content being cut. What could be eliminated was left up to the designers but those decisions were filtered through departmental concerns. The number of exams needed also conflicted with our design via content coverage as we had to use three two-hour class periods to accommodate our 
focus on assessing the students working in groups. Eventually we had to move to a night exam model due to our concerns about the time we were losing to examinations.

The use of homework and especially the use of pre-class homework also produced conflicts with our alignment with the COP framework. Both the pre- and post-homework problems are a mix of conceptual questions and traditional back-of-the-book problems. However, both types of homework questions are more directed and less authentic than the in class work, which reduces the opportunity for students to negotiate their learning goals and meanings for these activities. A bigger concern is the clear tension between using homework at all in a class that is subscribing to situated learning. There is a limited ability to make learning a social activity focused around enculturation with an online homework system. We do try and socialize the homework by making it a norm that the faculty and LA's occupy the help room for Friday's and Monday's when the pre- and post-homework is due, and students are encouraged to come as a group. However, there has been limited success with this approach. Anecdotally, we have also observed that students will meet up in their in class groups to do homework and prepare for exams, but again, we could not claim that this is a prevalent occurrence. We would argue though that the benefits of the preclass homework outweigh the tension in alignment with the COP framework. A prime example of this is the use of preclass homework to build scaffolds for computational activities. For instance, the second part of the problem illustrated in Fig. 3 is to build a computational model of a satellite rotating around the Earth. Students struggled immensely with the coding of the unit vector for the gravitational force until we started including preclass homework problems that scaffolded what a unit vector is and how it is used, combined with a question asking students to interpret lines of code about separation vectors. As we will discuss in Sec. XI, the research component of PBL had to be replaced in some capacity in order to lay the ground work for productive group discussion in the classroom. The prehomework acted as this replacement and is essential to achieving the goal of promoting discussion in class. In medical schools, the research component is an important practice that students need to develop in order to become expert diagnosticians. For students in introductory physics classes, we could not expect students to engage in research in a productive way without some form of scaffolding. We also could not expect all students to enter the class with the vocabulary of physics and so this is why the research component of PBL became preclass homework that invites students to prepare for class.

Institutional and subject norms also provide indirect conflict with subscribing to a COP framework to curriculum design. In running the class for over 5 years, it is clear that there is an observable adjustment period for students who are taking the class. Although we have not managed to study this phenomenon, it is clear that P-Cubed breaks students' expectations for traditional STEM class experiences. Because of the majority of students being STEM majors and having experiences typically with close-ended problems, the focus on open-ended problems where students negotiate together the end point, solution, and learning goals causes confusion. Students articulate a difficulty in knowing "what is important" or what to focus on in regards to preparing for an exam. There is a distinguishable difference in average score between the first exam and the rest of the exams students take in the class. This is true even now after working on the class for 5 years and implementing multiple strategies to address this problem. The question that might be asked is "why then have this exam?" The pressure from institutional norms and expectations meant exams were a requirement. Additionally, getting rid of the first exam would likely just result in the same problems occurring in the second exam instead because the emphasis on conceptual understanding rather than rewarding rote memorization would still be consistent. Also in relation to the open-ended problems, students initially struggle to negotiate learning goals and often misinterpret the in-class activities. Despite our intentions, there is a higher level of explicit guidance provided at the beginning of the semester to help students negotiate appropriate learning goals.

The student population is also another constraint on our design. Before designing the classroom, we were aware that the majority of students who would take the class would be first-year engineering and science majors. Since the students are typically in their first-year, this meant that our expectation around their current level of experience with scientific and engineering practices, and the progression we could expect in those practices, had to be set relatively low. This meant that from a design perspective, even though we aimed to provide some level of an accelerated trajectory for our students, we could not, for example, expect all our students to become expert problem solvers or planners by the end of the semester.

The need to assign grades and in particular the need to provide individual grades to students was the other major design constraint we had to work under. The P-Cubed class is an introductory physics class in a American university and is used as a prerequisite for entrance into the College of Engineering at MSU. Because of this context, summative assessment had to be inherently part of the class design. While students must be given numerical grades in the course as a part of the larger university system, the designers also wanted to include formative assessment in order to advise students where they are in a more detailed way and provide advice on how to improve and meet instructor expected levels. The decision to use formative assessment was also an attempt to align with the old timers and newcomers model of a COP (see Sec. XI E). However, 
the feedback that students receive is both formative and summative. Butler in the past has demonstrated that giving grades either with commentary or without commentary was less effective in improving students work than providing commentary only [91,92]. Dweck has also demonstrated that feedback that includes grades has long term negative impacts on students' perceptions of their ability to learn [93]. The proposed reasoning for this result is that students focus only on the grade and ignore the attached feedback. Given the results of this research, why did we then choose to pair our formative feedback with a score? It is important to note that formative and summative refer to "different purposes of assessment and not to different kinds or forms of assessment" [94]. We would argue that the score associated with the in-class feedback provides important information on where the students are in relation to expectations relating to scientific and engineering practices. For our students, expertise in scientific and engineering practices is hard to gauge due to their limited experience with these practices and the somewhat inscrutable nature of what it means to be "good" at them. We believe the score makes the practices a bit less inscrutable, which we believe to be important at this early point in their practice development process. We also believe the score aligns with their expectations of assessment at this time in their college career.

The Butler studies focus on interest and motivation; however, interest and motivation also relate to concepts like approaches to learning [95] or metacognition [96]. A student with a deep approach [95] to learning within a class will be intrinsically motivated to learn in that class. But there are multiple possible approaches to a class one of which is called a strategic approach which is were students choose between a deep or surface approach depending on their perception of what will result in the best outcome. These students can take a deep approach without being intrinsically motivated to do so like students who just take a deep approach and do not take strategic approaches to their classes. Additionally, the ability to choose an approach to learning within a class has been linked to metacognitive development. Part of developing metacognitively is that students have to learn how to give and receive feedback [97]. We are attempting to scaffold metacognitive development by giving a score that is associated with a detailed rubric. If students want to improve their score they either have to pay attention to the rubric which is built around scaffolding the development of scientific practices or the actual feedback they received or both. We would argue that the course learning goals are aligned with what the students are being assessed on for the in-class grade. So whether the students are paying attention to the feedback or the rubric they should still be receiving guidance in how to develop expertise in practice.

We also paired our score and feedback together in order to provide our students with different representations (both verbal and numerical) of the assessment of their understanding and practice competence. This aligns with principles highlighted in universal design [98] by offering students multiple ways to engage with the summative assessment in the P-Cubed context.

Finally, part of our decision process was influenced by our previous experiences in curriculum design using constructive alignment. If a major learning goal of ours was to develop students in their scientific practices, then it was important to align our summative assessment to evaluating students achievement of this goal. It is important to note that we are not necessarily disagreeing with previous research on providing the feedback and score together as being a harmful approach to assessment. We currently have no publishable evidence that would be contrarian to this claim. But we would argue that our approach is more nuanced and in a more complex environment than the studies outlined by Butler and Dweck. At the moment, the P-Cubed feedback approach is in no way fully understood, and we continue to conduct research on the process from various angles in order to understand the positive and negative impacts it is having on our students and teachers [99-102]. We hope to provide a more concrete understanding of its impact in the future.

\section{DESIGN DECISIONS}

In this section, we explain our decisions within our context and how it aligns with features of the COP framework outlined in Secs. IV-VII. Any curriculum design project will be contextually dependent. By outlining our design choices within our context, we can guide others on how they might operationalize the framework as an approach to curriculum design.

\section{A. Design decision 1-Decide on communities}

The authors of this paper have previously subscribed to the curriculum design approach of constructive alignment [13]. Constructive alignment refers to the alignment of learning objectives with the assessment methods, which are further aligned with the activities in which the students engage [103,104]. Typically we will design an activity or a curriculum by asking ourselves what learning objectives we want for the students. However, adopting the COP framework introduces an alternative set of initial questions at the outset of a curriculum design project. This is due to our desire for the design to focus on facilitating the development of COPs and our to need to negotitate between overlapping COP experiences. These questions include: Which COP or COPs will these students be practicing in? Is the COP related to another COP or if multiple COPs, how are they related to each other? And, within which of these communities do we want our students to experience a progressive trajectory in? Answers to these questions 
inform the majority of the curriculum decisions that we made.

Considering the majority of the audience for an introductory physics course for engineers will be engineers, then is designing the class to provide an inbound trajectory into the community of practicing physicists the best choice? Instead, we assume overlapping COPs to decide what we want to focus on as we believe that two communities (the community of undergraduate engineers and the community of undergraduate physicists) have shared practices. We also decided that in order for the experiences within this physics context to have meaning to engineers, then we should focus as much of our curriculum on scientific and engineering practice development as we do on conceptual physics development. This approach makes sense as professional physicists and engineers share several overlapping practices such as modeling, mathematical and computational thinking, and engaging in argumentation.

After deciding that these two communities are our focus, we then decide which of the overlapping practices, which are present and important in both communities, should be focused on developing in the course. In the case of P-Cubed, the shared practices include group work, decision making, facilitation, problem solving, modeling, and planning. By acknowledging that the development of these practices is a focal point of the class, we use constructive alignment to decide on what forms of assessment and activities will align with the development of these scientific and engineering practices (Sec. VII F). It is important to note that we are making assumptions by deciding which communities inform our curriculum development. We assume that the practices learned in one community will transfer to another. Furthermore, we have assumed that even though the communities are contextually different, engaging in these shared practices will result in positive trajectories in both communities. The literature highlights that having the opportunity to learn the knowledge, rituals, and histories within each community around shared practices will result in an understanding of the shared value that each community has for these practices $[57,69]$, which in part justifies these assumptions.

To mitigate the concern of whether practices were valuable in each community, we talked with faculty in the College of Engineering about the practice-focused change that we made to the introductory class for their majors. These meetings provided feedback on how the faculty in the College of Engineering valued these practices. In addition, the enthusiasm for the changes also provided legitimacy in messaging to the students that these are shared practices and essential to their subject-specific identity development. Our notes from the meeting indicated that the engineering faculty present highlighted the emphasis on group work, and in particular, on the improvement of group-based skills, which facilitate the development of effective groups - a high priority in engineering.
As previously asserted, we do not assume the classroom itself can be designed to be a COP. However, we made many of the subsequent design decisions with the implication that we are offering the opportunity for a COP to develop. Thus, the classroom is an opportunity for the students to embed a mesoscale COP (classroom COP) within a macroscale COP (community of undergraduate engineers) (Sec. IX A). Similarly, because all the work in the classroom is collaborative and the groups remain together for an extended period, a COP could also emerge in the student groups within the classroom (a microscale COP).

\section{B. Design decision 2-Decide on type of learning paradigm}

There is a multitude of learning paradigms that a curriculum designer could use in any curriculum design project. However, the decision to use the COP framework limits the choices available to some extent. The assumptions that we have made about learning and knowledge as part of aligning with the COP framework impact the design of the learning environment and how the target demographic of students interact in that environment [105]. As indicated in Sec. VI, constructivism is an influence on what it means to learn in the COP framework. This influence means that many of the design features of transformedbased teaching approaches still apply when considering curriculum development informed by COP. For example, there should be a similar move away from the transmission of understanding. Instead, the focus should be on a learning environment where the students are constructing their understanding with support from their teacher who is facilitating the learning. In the P-Cubed context, the learning is group-based and uses ill structured problems with negotiable learning goals $[22,24,106,107]$. For this context, ill structured means that a problem has multiple possible answers, is missing information (that may need to be assumed or looked up), and requires students to analyze, reason, and make decisions based on acquired knowledge and information. Negotiable learning goals means that goals may shift as groups navigate their solutions. The paradigm needs to change so the learner is working on authentic tasks that require the concepts and practices that we want our students to develop an understanding of how to use. "Use" being the operative word as opposed simply "know." As described below, the best attempt should be made for the activities to be authentic and based in the real world. In this instance, we define the real world as requiring the students to feel the same cognitive demands as if they had encountered the problem in a more authentic environment. Therefore, the activity should involve authentic problem solving, group work, decision making, facilitation, modeling, and planning.

From the COP framework and constructivist roots, we would be expected to incorporate several elements into a 
course design. Namely, instructors should be facilitating the learning, rather than transferring knowledge (Sec. VII D). Learning should be group based to facilitate mutual engagement (Sec. VII B). Activities should be ill-structured problems that provide opportunities for the use of practices and concepts in an authentic manner. These elements further limit the choice of available learning paradigms.

For P-Cubed, an adapted problem-based learning approach was the chosen learning paradigm as it traditionally incorporates the majority of these elements [108-110]. An adaption was needed to turn the focus from problem solving in an authentic context to include a focus on many practices. This adaption was accomplished through the problem design as described below and through the feedback-based assessment (Sec. XIE).

Traditionally, a problem-based classroom does not have a lecture component, and instead, the focus is on identifying learning goals and doing research in these areas to be able to answer the question between problem-solving sessions [111]. Being able to engage in productive research in order to prepare for class is not a small ask of students, especially introductory students. The act of identifying learning goals by itself is a vital part of the practice of analysis, which is considered a higher-order thinking skill [112]. Yet, students needed enough grounding in the ideas presented by a problem in order to engage productively. An option to provide this grounding could be a lecture component. However, including a lecture component to the class would provide a counternarrative about how knowledge and learning occur, which would not be in line with the learning theories to which we have chosen to subscribe. Instead, we incorporated flipped aspects to the course [113]. Here, the focus is on pre-homework that should be completed from information gained through the watching of videos, reading of notes, or completing of research in order to prepare for class. The videos and prehomework also act as scaffolds for the students to identify what the essential learning goals are for the next week of class.

The preclass preparation does set up some of the conditions for a COP: mutual engagement (Sec. VII B) and near-peer guidance (Sec. VII D). Without preparation, it is difficult for the students to communicate about shared topics. Furthermore, the need for preparation builds up the sense of coresponsibility. Preparation can also act as a way for a student to become a near-peer guide as they prepare to the point of having more insight and practice experience. From a messaging perspective, we communicate that preclass homework is a way to obtain some initial vocabulary, knowledge, and practice before it is developed more thoroughly in their groups.

\section{Design decision 3-How do we design for authentic activities?}

Within the classroom, authentic activities provide the motivation and context for students' development of practices within the macro-scale community (Secs. VII F and VII E). For the P-Cubed context, authentic activities mean a problem or dilemma that is reflective of what a practicing physicist or engineer might encounter. We show an example of an in-class activity from P-Cubed in Fig. 1. In this activity, we task students with placing a satellite in geosynchronous orbit around the Earth.

In designing this style of authentic activity, the first and foremost feature is that the activity prompt is ill structured. For example, in the geosynchronous orbit problem in Fig. 1, students are not told the altitude at which a satellite

\section{Project 3: Part A: Geostationary orbit}

The Carver Media Group is planning the launch of a new communications satellite. Elliot Carver (head of Carver Media Group) is concerned about the launch. This is a $\$ 200,000,000$ endeavor. In particular, he is worried about the orbital speed necessary to maintain the satellite's geostationary orbit (and if that depends on the launch mass). You were hired as an engineer on the launch team. Carver has asked that you allay his concerns.

\section{Project 3: Learning issues}

- Forces cause changes in momentum

- Uniform circular motion

- Newtonian gravitational force

FIG. 1. This is the activity prompt for one of the authentic activities used in the P-Cubed classroom. This activity is expected to take a group of four students two hours to complete. 
is geosynchronous or even provided a definition of what geosynchronous means. Instead, they are given an end goal and expected to decide as a group how they will acquire any missing information.

Ill-structured and underdefined problems are used intentionally for several reasons. First, it is authentic to the tasks required of professional physicists and engineers. In professional practice, a physicist is not given a well-defined problem with only one missing quantity that expects a single numerical answer. Thus, we do not start with simplified, unrealistic problems because this would not be reflective of physics practice. Rather, we create problems that reflect the complex and intricate issues that hold together the physics COP. Furthermore, these problems reflect the complexity of the thinking and work that students are expected to be able to do outside of the school context when this learning is complete. Not only are the problems helping students develop their practices within the macro-scale physics community, but they are hopefully developing the skills that will be transferable to their other courses and future careers.

Additionally, when problems are underdefined in this way, students can impose their problem frames on them $[114,115]$. Thus, students can place their own framing on the problem and as a consequence take ownership of the process. Within their group, students must make decisions about how to simplify and solve such a complex problem. In doing so, students are defining and creating the particular problem that they will solve. This decision-making process also means it is always possible to work a little longer in an attempt to develop a different rationale for a solution, to design a more complete solution, or to consider better alternatives. It is in this inquiry into ill-structured dilemmas that ownership and learning occur. An example of learning that occurs is the students appropriating practices such as modeling and problem solving. The students have to decide what it is they are modeling or solving and what approach they should take to creating the model. They also have to make a group-based decision about the model or problem solving approach - the decision itself is another practice. Each of these are practices that we want students to engage with in order to renegotiate their identity in both local and global communities.

Under-defined and ill-structured problems also promote negotiation, collaboration, and mutual engagement between group members (Sec. VII B). These problems are not intended to be completed alone. Instead, the work is collaborative and social—continually occurring through a process of negotiation within the group. The tutors guide the quality and the depth of this negotiation, and the understanding of the content and problem to an extent. But because it is a social environment they test their understanding by incorporating the issues, points of view, and understanding of others $[24,116]$. The importance of a learning environment that emphasizes discussion and building concrete understanding is critical to the design of a productive learning environment centered on practices [117]. Thus, the lack of definition requires students to explain their understanding to their group members. This leads to a negotiation about the problem goals and solution amongst the group, which promotes students' mutual engagement and understanding from the problem.

An extra element of authenticity that we have integrated into the classroom is the use of computational modeling to solve physics problems. The critical reality for both physics and engineering majors is that there is a high probability that programming will have some involvement in their professional careers. We introduce computation in a way that parallels our ill-structured problem-based learning activities in the form of minimally working programs [33]. The approach of minimally working programs transforms computation into a collaborative exercise that is in line with the COP framework.

Ill-structured and underdefined does not mean that students are left to complete the problem on their own or to change the premise of the problems completely. That is, learning issues cannot be solely self-determined by the group. Instead, we need to introduce students to the community and the issues or problems that engage that community. In part, this is controlled by the role of the teaching staff in the classroom. We discuss the roles of staff below. However, this is also, in part, addressed through the context of the problem. The relevance of the problem to our class COP and the context of the problem must be at the forefront when we introduce students to it. This introduction should be in a way that challenges and engages students [24]. In the problem given in Fig. 1, students are learning the content goal of orbital motion and Newtonian gravitation while also engaging in practices such as problem solving and modeling. While we could have chosen any number of contexts for this learning goal, the geosynchronous orbit allows students to investigate a context that engages and opens the discussion to the relevance of geosynchronous satellites to our society. Through this problem context, we are making the problem relevant to the learner [118]. We are also engaging students in a problem that is both relevant to their lives and relevant to the physics community.

\section{Design decision 4-What is the role of the various teaching staff?}

While problems are ill structured and underdefined, this does not mean that students are left to negotiate and enter the COP on their own. The instructors play an essential part in both scaffolding the problems and facilitating work that promotes development in the practices of the community (Sec. VII D). In P-Cubed, there are a variety of instructors in the room, typically one faculty member, one graduate teaching assistant (GTA), and 10-12 undergraduate 
learning assistants (LAs) for a classroom of 100 students. While the faculty member and GTA are typically from the physics department, the LAs come from a wide variety of majors in science and engineering. Within the P-Cubed course, all instructional staff are referred to as "tutors" because they have the same role when interacting with students in the class. Each tutor is responsible for facilitating 2-3 groups of students (8-12 students per tutor).

Because each tutor is only working with 2-3 groups, the tutor acts as a facilitator for those groups. The tutor role of a facilitator takes on a specific meaning within the COP framework $[22,119,120]$. The role can manifest in different forms including a moderator, a coach, or a role more aligned with the COP framework: mentor [121]. The instructor, at times, may act as a group leader to model the expectations of a leader within a group. At other times, they should be a facilitator who gently nudges the direction or content of discussion and learning towards the learning goals of the problem [120].

Johnson outlines the duties of the instructor to be readying or guiding discussion around the following three points [22]: (i) Goals and criteria for meeting goals, (ii) Evaluation of whether the students are meeting the goals, plus (iii) Peer evaluation and self-evaluation. Within P-Cubed, we implement this approach in the training of the teaching staff and the role that the tutors take in class. As Palloff and Pratt discuss, the tutors are there to help the groups through their activities, not to provide answers or "transfer content" [120]. Squire and Johnson support this idea that the facilitator role, if implemented successfully, should be more valuable than a content provider or source to answers to questions [122]. Instead, tutors are asked to help students by asking them questions or helping them focus on particular areas in the problem. For instance, if a group was struggling with how to start the geosynchronous orbit problem (Fig. 1), the tutor might ask the group questions to direct them toward creating a goal as a group. Alternatively, they might ask a question to help the group identify what they know and what they need more information about in order to solve the problem. This motivation returns to the idea that authentic activities should include student ownership in the problem-solving process.

In a facilitative role, tutors are there to help students explore their ideas rather than telling them how to solve the problems. Tutors do choose to promote or cutoff ideas depending on situational factors such as the group's progress and the time left in class. The tutors' teaching practice in this respect points to Wenger's educational alignment as well as the need for students to understand how to become effective in more central practices (Sec. V). As identified by Powers and Guan, students who identify self-motivating factors and self direct their learning will be more motivated than those who experience a lecture-based teaching style or content transfer transmitted between the instructor and the participants [123]. Thus, in P-Cubed, the tutor's job is to coach and to model learning by asking the questions the students should be asking themselves. Tutors are not participating as members of the group. Rather, tutors aim their interactions at moving students towards developing expertise in asking productive questions rather than getting the "right" answer [24,124].

Because the P-Cubed classroom consists of a community with different levels of expertise (faculty members, GTAs, and LAs), this style of facilitation allows two aspects of collaboration to develop: peer interaction and expert-toapprentice interaction. These interactions are particularly interesting with regards to the LAs in the course because they can facilitate both aspects of collaboration. In the context of COP, peer interaction is a fundamental enabler of the COP by facilitating negotiation and discussion [119]. Negotiation and co-construction allow for the community to transform collaborative efforts into "artifacts" (e.g., symbols, procedures, rules, technology, and products) [5] (p. 52). According to Wenger, the same process also accounts for the development of shared history through negotiated meaning. LAs can function in the role of a peer or near peer because they are also undergraduate students and have recently taken the course. We generally distinguish "peers" as students who are taking the class and "near peer" to be the LAs who are facilitating the class, but that distinction is dependent on students' perceptions. However, LAs are also promoted as experts in the classroom because they are tutors for 2-3 student groups, which is the same teaching load as a GTA or faculty member. In the COP framework, the LAs offer a pathway into the communityproviding a bridge between the periphery members and the central members. This bridge sets up a model for students where LAs (who are near peers with students) are central members of the classroom community. The LAs connect to the GTA and faculty members who are more central to the community of practicing physicists.

\section{E. Design decision 5-Trajectory guidance: Feedback}

Feedback as a curriculum tool has been demonstrated to enhance achievement, particularly in contexts where it is being used to facilitate the development of professional practices or skills $[37,125]$. From the perspective of situated learning, feedback is an opportunity for more central members of a community to provide newcomers with insight into the more central practices and the shared repertoire of a community (Sec. VII D) [37]. Feedback lays out a path for how to develop expertise in the practices of the community. In essence, the feedback can act as a tool for enculturation into a community. It can also act as a tool for communicating to students how to negotiate membership in multiple COPs with shared skills and practices at the same time. The power of feedback can be amplified by having undergraduates who are and have negotiated multiple memberships in the past being the communicators. 


\begin{abstract}
Hey all, this group is already functioning really well, and I appreciate the excellent collaborative atmosphere that you all have fostered in this short time. You really adopted some of the norms we are trying to encourage, like using the four quadrants and developing a summary answer at the end of each part of an activity. From an improvement perspective, I want to build these norms further and push you to improve on them. For the four quadrants, I think making sure to focus on the assumptions and approximations is the next step in improving your physics problem-solving. These are so fundamental to doing physics, so make sure to record and have conversations around the assumptions and approximations for each part of the problem. Just keeping asking yourself what did we do that is unrealistic, and you will find yourself identifying the assumptions and approximations you explicitly made and maybe some you didn't realize. Adam, I enjoy and appreciate the energy that you are bringing to the group. I think your positivity is infectious and has helped the group make progress, especially when you all have become stuck. Having positivity when something becomes difficult can be tricky, so I hope it continues as we move into the next set of activities. An aspect I would like you to work on is to listen more actively. Listening more actively can mean many things, but for a scientist or engineer, I think it means taking another person's idea and highlighting how it fits with your thinking as opposed to just highlighting how it does not fit. I found on Thursday of last week that it took you a while to take on another's perspective, and this can often be because you are focused on how it doesn't fit as opposed to how it does match. Don't get me wrong I still want you to disagree, but I want your disagreements to be constructive and help you build on each other's ideas.
\end{abstract}

\title{
Group Focus: $\mathbf{9 0 / 1 0 0}$
}

Group Understanding: $90 / 100$

Individual Understanding: 95/100

FIG. 2. An example of feedback provided by a P-Cubed tutor to a student, which highlights both the structure of the feedback and the corresponding scores for the student.

Feedback in a classroom can come in many forms, both formative and summative. The distinguishing feature between the forms is that the aim of formative feedback is to provide meaningful advice on how to improve. By contrast, summative feedback is meant to evaluate student achievements. It can come in the forms as simple as communicating the correctness of an answer. In the P-Cubed model, students are given individualized formative feedback each week. The role of feedback in P-Cubed is to outline to students how to become better aligned with professional practices that apply to both the undergraduate physics COP and the undergraduate engineering COP. The feedback in P-Cubed threads a complex line of being critical, generous, challenging, and respectful at the same time.

The feedback should be focused not simply on formative content, that is, what practices the student needs to develop and how they can do so. The approach to feedback should also be designed to build an atmosphere of trust within the community. Developing trust between the tutor and student allows the feedback to influence students' attitudes about the practices of the community. It should also bolster the perception that the students are receiving guidance to central practice, even when it comes from a LA. In P-Cubed, the weekly feedback is a form of formative assessment that explicitly focuses on the practices of the individual and then the group as a whole. The feedback is both affirmative and corrective, with students receiving praise for conducting a particular practice well—based on observations of their work in class. The feedback also highlights an aspect of their work with a given practice that they should consider correcting in order to improve in that practice [100].

The feedback mechanism is communicated to staff and LA's via training, and they are provided a framework that is based off the previous discussed points. The instructions given to the teaching staff are to highlight a positive, identify a possible improvement and then suggesting a strategy for this improvement. This three-part cycle is intended to be completed for both the group as a whole and for the individual. In the feedback example presented in Fig. 2, two positives are highlighted for the group-they have built an atmosphere of collaboration and they have adapted to P-Cubed norms in the form of summary white boards and the four quadrants. A possible improvement is highlighted in the form of developing their ability to identify and discuss approximations and assumptions. Finally a strategy is proposed which is to "just keep asking yourself what did we do that is unrealistic?" The three partcycle starts again for Adam individually when a positive is highlighted as his "positivity is infectious and has helped the group make progress." Next a possible improvement is suggested "to listen more actively," and then a strategy to develop in this area is highlighted in the form of "taking another person's idea and highlighting how it fits with your thinking as opposed to highlighting how it does not fit." Elements of the tutor (who wrote the feedback) trying to develop trust in the environment are highlighted by the identification of "the excellent collaborative atmosphere" and also trying to ensure disagreements are constructive and not destructive. The scores Adam received also align with the positive description of the groups progress and that Adam is in particular doing well in regards to his own understanding. The rubric for individual understanding highlights the role of disagreement and in particular justifying disagreement with physical reasoning. Thus, we see alignment between the feedback and score since Adam has demonstrated this in his group interactions for the week.

Including the feedback mechanism within P-Cubed was an essential curriculum design decision to align with the 
COP framework. It is an explicit attempt to provide the central guidance inherent within a COP on a frequent basis, and it acts as a way to reinforce the students' legitimacy in their practice. The feedback mechanism is also a form of summative assessment, as we give individual students a grade associated with their interactions in the previous week.

We believe this feedback is a form of authentic assessment (Sec. VII F) because of its alignment with practices both in the undergraduate communities that the students are members of and alignment with the professional practice of engineers and scientists. Past students who have entered professional science or professional engineering environments have indicated that this assessment aligns with professional development approaches in industry. We divide the rubric for the assessment part of the feedback mechanism into three categories: individual understanding, group understanding, and group focus. The development of a sense of coresponsibility is a centerpiece of the assessment as demonstrated by the focus of two of the three categories on group-based topics. This focus stemmed not merely from trying to build the condition of coresponsibility for a COP within the student groups, but also from a concern that emergent leaders within groups can sometimes control the quality of learning of their other group members through the decisions they make for their group.

Another framing for the feedback is its ability to counter the process of disidentification or where students begin to disengage in participation. Disidentification frequently refers to when students are engaged in ways that appear to be going through the motions of the practices of the COP, but they are withdrawing from identifying with the community. Withdrawal relates to concerns brought up by Johnson about the role of unmotivated members of the community when the COP framework is applied to the classroom setting [22]. The feedback can act as an intervention for when students are observed to be not fully participating in the community and not working on developing their expertise in the practices of the learning environment. It can be used to affirm that aspects of their practice are legitimate and help negotiate the legitimacy of the physics practices in the engineering context. A concern of applying COP to the classroom is the questionable authenticity of the teacher being the central member of a community. The source of the concern is that the teacher has to bridge the large gap between their expertise and the expertise of their students [22]. We have already indicated the role LAs play in reducing the size of this gap. We believe this argument holds when transposing it to the feedback mechanism. The LAs provide feedback on the practices of the community as filtered through their interpretation of the practices, which further reduces the gap that needs to be bridged.

\section{F. Design decision 6-Cultural artifacts}

Criticism of the application of the COP framework to the classroom setting has previously focused on the lack of a preexisting community to join [24,126]. An argument for this criticism is that each new implementation of a class resets any cultural development that has occurred as the members move on to new communities. However, there is no specific time constraint or any known impact on a COP existing for a specific length of time. The emphasis is on there being culture to appropriate. From a curriculum design perspective, the decision to place the classroom in the more expansive communities of undergraduate physicists and undergraduate engineers should mitigate this concern. There is an immense amount of physics and engineering culture present in the classroom in the forms of language, jargon, representation conventions, terminology, and conceptual artifacts. The inverse concern could be valid. That there is, in fact, a large swath of possibly impenetrable cultural practices and discursive conventions for students to appropriate in an introductory physics classroom. We would argue that the focus of the facilitation by LAs might again mitigate the impenetrability of these cultural components of the class. However, students in the classroom will have little impact on the culture at the scale of the undergraduate COP. Instead, it is at the class level where culture can be built collaboratively and negotiated by students.

To tackle the cultural scalability problem, we made a design decision in the initial implementation of the class to embrace the idea of developing local lore, jargon, and jokes that the students could evolve. We weaved a narrative throughout the analytical and computational problems that included the creation of a creature called the "boar tiger," which quickly became a mascot and source of amusement for the class (Fig. 3). The boar tiger quickly evolved into a cultural artifact that perpetuated to the point that there is an official boar tiger day each semester where the LAs all wear boar tiger t-shirts. Staff and students alike place boar tiger stickers on their laptops and water bottles. Boar tigers have made appearances around the bars and coffee houses of Lansing. As part of one of our initial reviews of this paper, a reviewer commented that they were unconvinced of the importance of the boar tiger in illustrating COP course development. We would argue that although it may be unique and be perceived by outsiders as frivolous, to the students in the P-Cubed community, it is a cultural marker of membership. Knowing what it is and how it relates to

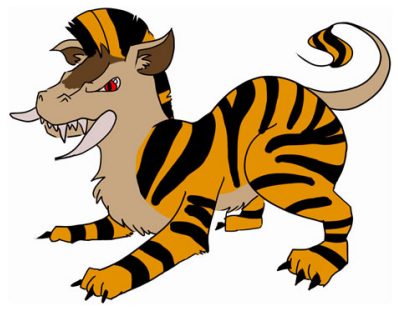

FIG. 3. The boar tiger, which is used as a cultural artifact in P-Cubed. 
physics means they have been or are a P-Cubed student. We highlight the boar tiger because it was negotiated to be a cultural marker by the students in the community and illustrates the importance of not conceiving culture within a physics class as just being physics orientated. It also highlights that a curriculum designer can include cultural artifacts that are ready to be negotiated by the students.

The narrative baked into the class around the boar tiger often includes absurd elements or humorous references to the teaching staff that can be edited at the beginning of the semester-depending on who is teaching the class. The narrative allows students to make decisions about the direction of the story culminating in the last week of class when students get to design their own problem. The "design your own problem" is a week-long event that we use as a review at the end of the semester for the concepts covered in the course. During the design-yourown problem week, we task each group with designing a problem that is in the same style as the authentic activities used all semester. Then a winner is chosen collectively by the tutors, and the whole class solves the winning group's problem in the next class session. In this way, students have the opportunity to impact an essential cultural element of the classroom. In the design process, the students can decide to contribute to the narrative developed in the class or to go in a different direction. On average, at least half of the groups incorporate the boar tiger or other previous narrative elements in humorous ways in their problem design. The narrative, jokes, and local lore allow for a preexisting culture and history within the class that offers students the opportunity for enculturation.

A different class-based approach to offering opportunities for reification was the introduction of flexible supports that a group could adapt per their needs. There is a two white board system promoted early in the semester, which introduces the students to the idea of having one board as an organization tool that is split up into four quadrants (Sec. V): facts, lacking, representations, and assumptions and approximations. The students then use the second board for rough work and calculations. Over time, the students decide how to use the two white board system and can decide to creatively adapt the four-quadrant system to include new quadrants or a completely different emphasis. Group white boards also become a resource that reflects the shared experience of the group and the reification of their work. Students integrate this work into the community through photos of the white boards.

\section{G. Design decision 7-Cultivating our messaging}

By messaging, we mean the communication that tutors provide to the students around the learning goals and curriculum decisions, which inform what students are doing and why they are doing it. For the P-Cubed classroom, we deliver this messaging in two distinct ways. The first form is an introductory presentation at the beginning of each semester that outlines the class structure and the reasoning behind that structure. During this presentation, we make explicit connections to the practices that are the focus of development in P-Cubed and how they connect to professional practice. This communication about this connection is our attempt to connect the potential COP in the classroom and the undergraduate community of physicists and the undergraduate community of engineers to some broader community. In this case, it is the communities of professional practice. The presentation creates a shared understanding of possible shared interests or desires that the students can pursue together as a community (Sec. VII A).

These shared understandings and interests are then reinforced by the second form of messaging. This second form of messaging is through the feedback that the tutors provide the students each week. As part of the feedback training process for teaching staff before they begin teaching P-Cubed, we emphasize connecting the students' practice in the classroom to professional environments or personal experiences. For example, we ask tutors to connect the need to develop group-based facilitation skills to project work in professional practice in science and engineering as most students will inevitably work on a team. We explicitly make these connections as an attempt to legitimatize practice. Furthermore, making these connections between practice in the classroom to something more significant is outlined by both the global or local duality and the need for the COP to be part of something more substantial (Sec. VII E).

\section{H. Design decision 8-group design}

The students in P-Cubed work together in groups of four for periods of between four and six weeks, depending on the exam schedule per semester. After each exam, the groups are split up, and new groups are formed. This is done so that new learning or cultural developments will be dispersed from small groups to the community at large through the distribution of individuals. We made two specific design decisions concerning the groups that are in line with the COP framework. The first is that the initial groups that we form at the beginning of the semester are designed based on students' responses to a survey delivered on the first day of class. Students describe their previous physics, math, computation, and group work experience on this survey and their perceived competence in each of those areas. Then, we distribute students into groups so that there is an expert in each of these capacities at the beginning of the semester. This decision aligns with new members of a community working alongside more competent members acting as potential guides in their practice.

After these initial groups, the new groups are formed based on the tutors' experiences with individuals. Typically, we create groups with at least one student facilitator, who will act as the more competent central member of the community. A student facilitator can both be an emergent or formal role 
that is encouraged. The role is characterized by a willingness to engage with the group-first mentality of the P-Cubed classroom and to act in a way to ensure full group participation as well as the development of a shared understanding. Facilitation is key to our approach to group work in P-Cubed (Sec. XID). Emergent leadership is an inevitability in group work, and we aim for only one type of leadership in P-Cubed - the facilitator [101]. The origin of this aim is to lean towards no individual leader in a traditional sense and instead focus on students taking up the role of facilitators. Individual leaders may emerge due to a vacuum of power, but the power they take up when they do become leaders can have consequences for the learning of the other group members. This is why we focus on fostering facilitation that tries to instill equality into the learning of all of group members. The fostering process occurs through both in-class interactions and feedback where facilitation-based behavior is highlighted or described. This focus on facilitation aligns with the approach of PBL. It also creates a collaborative environment of trust and shared decision making that helps the students in the development of practice.

The second curriculum decision around group design was to include group exams. Again, this decision highlights the collaborative nature of the classroom and promotes the idea of coresponsibility between group members. Group exams align with the idea of mutual engagement from the COP framework (Sec. VII B). The group exams account for $25 \%$ of students' overall exam grades and provide another opportunity to assess the students authentically (Sec. VII F). This decision aligns the assessment with the group-based practices that are the learning goals for the class. It also encourages the individual groups to develop a positive dynamic and group flow-essentially a bond-in order to be successful in the group exams, which aligns with the ideas of mutual engagement from the COP framework. Again our messaging around the group exams is an integral part of the curriculum element. Teaching staff make sure to communicate that if you are going to work in groups in class and if you are going to work in groups in professional practice, then at least part of the examination process will be in groups, and you will be rewarded if your group has built up the requisite understanding and practice.

\section{DISCUSSION AND CONCLUSION}

The assumptions and design decisions that we made and the context we had to navigate inevitably foreground certain aspects of the COP framework and background others. We feel that this is inevitable in attempting curriculum design from this perspective. We did our best to be explicit about what we are foregrounding in our assumptions and decisions. We also hope that the outline of COP with examples for learning theory aspects and conditions will inform those wishing to foreground different aspects or who have different contextual barriers to navigate.
We have presented the major design features of our P-Cubed classroom through a discussion of how those decisions align with the COP framework. We can not argue that all of these elements are prescriptive as we have not completed enough research to be able to claim the importance of one decision over the other. The developers of the COP framework have stated the three features of "shared interest or joint enterprise, mutual engagement and shared repertories" are essential for a COP to exist [5]. By extension, we can assume that any decision that impacts one of these elements could be important to providing the opportunity for the development of a COP. The "opportunity" is an important distinction to be made here. We are not assuming that a COP will form within the classroom every time, instead the assumption we are making is that we can provide the opportunity for one to develop and influence the community that develops in a positive way. Even if it does not form, the students are still engaging in peripheral practices of their discipline-based identities. In the past, attempts to purposefully design COPs have faced criticism for losing sight of the spontaneous nature of COPs [70]. Designing for the opportunity negates this criticism as spontaneity is still required for the community to develop. Below, we will outline common criticisms of COP based curriculum design and how they relate to the P-Cubed classroom. We will conclude by discussing previous research and future work in P-Cubed, along with other curriculum development projects inspired by COP.

\section{A. Common criticisms of COP based curriculum design}

A common criticism for curriculum design based on the COP framework is that the focus on teaching shared practices between the engineering and physics communities still means the practices are embedded within one community. The argument is that this embedded nature alienates learners from full experiences in those practices and results in contrived identities [11,24,127]. A counterargument that we have not yet been able to study but hope to in the future is that the focus on shared practices facilitates an ability for the communication between engineers and physicists to be easier. Of a similar theme, the authenticity of shared practices is questionable when the class is embedded in one community but attempting to develop practices that are common to two [11,127]. This even applies to authenticity within the community the learning is centered in, as the level of authenticity is not on par with activities that are integral to the ongoing goal of the professional community [24]. This can contribute to a contrived identity or limit the amount of discipline-based identity that could develop.

With respect to P-Cubed, these are fair criticisms of the model we developed. Our response is that we are not arguing for a large building of identity from the students' experiences in the P-Cubed classroom. We are, however, providing students with a taste of authentic group work, 
decision making, facilitation, problem solving, modeling, and planning. We aim for this taste to act as a scaffold for future development in these shared practices in their future discipline-based classes. Designing using the COP framework allows for a more authentic experience than a traditional approach to teaching introductory physics, but it cannot be akin to professional practice because of the peripheral nature of the concepts being dealt with in the class. The number of concepts that have to be covered is a limiting factor in any introductory course's ability to design the activities as being authentic, but that does not mean that the activities cannot involve authentic practice. As Irving and Sayre argued, creating an introductory course that is crucible in nature so that it provides an accelerated trajectory is questionable when introductory students are often learning the cultural norms of college at the same time [50]. Examining the transfer of practices from a physics context to an engineering context as well as how applicable those practices are in each different context is an important area to study in the future. We need to affirm that the focus on shared practices provides a noticeable impact on future practice in either the engineering or physics domain.

Another criticism of the COP framework as applied to the classroom context is that classrooms are often competitive environments that restrict the amount of collaboration that can occur [70]. Similar in nature, classrooms are often environments that have time pressures associated with them. This pressure reduces the emphasis that will be placed on developing a collective understanding [70]. Again, we believe these are legitimate concerns when it comes to the practical application of the framework to the classroom context.

In the P-Cubed environment, we highlight in our messaging at several times during the semester that the grading is not on a curve. The grading scale is fixed, so that in principle, every student could earn the highest awarded grades. We message that the focus in P-Cubed is, in fact, on collaborative effort. We further communicate this message through our in-class assessment that focuses on evaluating students' ability to collaborate. This same assessment focuses on group understanding, so again, we emphasize developing a collective understanding through the assessment. Messaging plays an important role in developing an atmosphere of trust and coresponsibility. The tutors frequently communicate that it is important to develop a collective deep understanding of the concepts and practices being covered as opposed to finishing the problems to completion or finishing the problems quickly.

There is also a discussion about variation in starting point and the inconsistent trajectories of students. No learning environment guarantees learning goals and the same has to be true for identity development. For our P-Cubed classroom, we have students coming in with different levels of understanding, experience with scientific practices, and subject-specific identity that may put them at different starting points on the periphery of the undergraduate physics and engineering communities. In our opinion that is okay. The important thing is to offer opportunities for a COP to develop. It is partially up to the students to take up that opportunity and engage authentically in the learning environment. If different trajectories occur, then this might be a natural side effect of the classroom design mixed with student backgrounds and experiences in overlapping communities. Nevertheless, it would be worth examining students' identity development associated with their experiences in the classroom in the future. This examination might be especially fruitful from the lens of the designed and emergent duality which might allow us to understand how much of their trajectory is a result of what was planned for and what emerged naturally in the learning environment.

Finally, a criticism of the COP framework that impacts curriculum design is its lack of theorizing of the role of power in learning environments $[12,128]$. Power and who has it is imperatively important in any learning environment. Lave and Wenger mention the significance of power and its influence on the legitimacy of peripherality and participation, but they have not explored this influence in any great depth [7]. Distribution of power in a classroom, especially in a group-based classroom, is important to understand. As mentioned previously, a concern of turning over the learning to groups of students is that emergent leaders can control the quantity and quality of what is learned. An example of this control is deciding on a goal of getting a solution as quickly as possible and ignoring their group members lack of understanding. We have made initial steps to understand leadership in the context of the P-Cubed learning groups but are still in the beginnings of this research [101]. It is important to continue in order to understand power dynamics within groups in this style of learning environment.

Within the tutors as well, there are potential generational power struggles between instructors and GTAs, GTAs and LAs, instructors and LAs, or even instructors and P-Cubed designers [70]. Essentially, there is potential conflict between old-timers and newcomers in many membership groups. Evidence of this conflict can be found in Pawlak's examination of teaching approaches in P-Cubed with older generational LAs having different approaches to teaching computational problems than LAs who are relative newcomers in comparison [129]. This lack of focus on power within the COP framework is a shortcoming. There is a need to further understand the complex power dynamics at play within the P-Cubed environment.

\section{B. Alignment of COPs}

Design decision 1, which focuses on choosing the communities you are designing for, opens up an important question as to whether the practices of the undergraduate COPs of physics and engineering actually align with professional practice within those areas. Work by 
Tonso [130] and also by Seymour [131] has highlighted that STEM undergraduate programs are often the antithesis of STEM professional practice. We would argue within our context the practices central to the communities of undergraduate physics and engineers are aligned to a degree with professional engineers and physicists. We have evidence of this in that the physics department recently changed the major program to include a computational requirement for physics majors. Additionally, in our discussions with engineering faculty, they emphasized the development of teamwork as the most important learning goals that are applicable to their engineering majors from the perspective of their future engineering classes. However, this is not to say that all the classes the students will take in the context of MSU engineering or physics align with professional practice. The reality is that we are sourcing both professional and undergraduate COPs for the practices we are focusing on developing in our class, but the level of authenticity with which we can inject the practices is more akin to undergraduate COP. However, our class is intentionally aligned with professional practice as that is where the majority of students who take our introductory course are destined to end up.

\section{EMP-Cubed and studio COP designs}

The second class in the introductory sequence of P-Cubed was constructed in 2017 and called Electricity and Magnetism Projects and Practices in Physics (EMPCubed). P-Cubed had already been running for three years when the development process for EMP-Cubed began. EMP-Cubed incorporated nearly all of the same design decisions with one exception. The feedback system is adapted to switch from the tutors giving feedback to students to a self-feedback model halfway through the semester. Students provide themselves with feedback that is iterated on by the tutors [132]. This is an attempt to begin to scaffold the transition to central membership. Central members become more reliant on internal guidance to their learning through lifelong learner skills rather than external guidance by a more central member.

We have also applied the COP framework to a studiostyle classroom instead of a PBL paradigm. The studio style is perhaps even more aligned with instituting the framework due to large group discussions being an essential part of the constructed knowledge in the community. The inclusion of discovery-based experiments and formal laboratories also allows for access to more authentic practices. However, it is also open to same criticism with respect to the level of authenticity of such experiments from the perspective of what central members do in their day-today practice. The experimental component also opens up questions of whether experimental skills are a shared practice between the physics and engineering undergraduate communities. Applying the COP framework to another paradigm of teaching does allow for investigating the frameworks applicability to the physics from another perspective.

\section{Future research to inform using COP as a design principle}

In Sec. IX D, we discussed how different classes can provide opportunities for different trajectories. In order to provide a narrow, succinct scope to this paper, we focused our discussion on the implementation of COP to a single introductory physics course. Instead, we could ask ourselves what a whole curriculum designed via a COP perspective could accomplish. What if certain classes could be designed to combine together to provide the opportunity for solidifying a STEM identity or a physics identity?

We will admit that the application of the COP framework to the physics introductory context brings more questions than answers. In previous work, we have demonstrated typical gains on the FMCE for a transformed class and slightly positive movement on the CLASS survey [13]. Furthermore, we have presented evidence of the development of group work skills through interviews and investigated LAs approach to teaching computation in the class $[13,129]$. We also have investigated the impact that giving feedback has had on the LAs in the class, which has demonstrated that for at least the LAs, there is an ability for them to transfer practices between contexts [99]. However, identity development and practice development are not easily measurable outcomes. In fact, development in these areas might not have an immediate or predictable impact on the students. It may take comparative experiences in other contexts for students to understand the development they have experienced. Because of the nature of these developments and the complexity of the learning environment constructed, it is important that we continue to study the impacts of designing a introductory physics classroom informed by the COP framework.

In closing, we have laid out the design decisions behind the construction of the P-Cubed classroom and how they were informed by the COP framework. By being transparent about our decision making process and making explicit the connections to the underlying theory, we hope to inform others who are interested in designing classrooms aligned with the COP framework.

\section{ACKNOWLEDGMENTS}

This work has been financially supported by the CREATE for STEM Institute (funding for curriculum development and staffing) and the Department of Physics and Astronomy (learning assistant support) at Michigan State University. The authors would like to thank Eleanor Sayre, Paul Hamerski, and Michael Obsniuk. The authors would also like to extend a special thank you to all of the LAs, TAs, and MSU staff who have taught in the P-Cubed class. 
[1] E. W. Close, J. Conn, and H. G. Close, Becoming physics people: Development of integrated physics identity through the Learning Assistant experience, Phys. Rev. Phys. Educ. Res. 12, 010109 (2016).

[2] I. Rodriguez, R. M. Goertzen, E. Brewe, and L.H. Kramer, Developing a physics expert identity in a biophysics research group, Phys. Rev. ST Phys. Educ. Res. 11, 010116 (2015).

[3] A. T. Danielsson, Exploring woman university physics students 'doing gender' and 'doing physics', Gender Educ. 24, 25 (2012).

[4] P. W. Irving and E. C. Sayre, Conditions for building a community of practice in an advanced physics laboratory, Phys. Rev. ST Phys. Educ. Res. 10, 010109 (2014).

[5] E. Wenger, Communities of Practice: Learning, Meaning, and Identity (Cambridge University Press, Cambridge, England, 1998).

[6] N. W. Brickhouse, P. Lowery, and K. Schultz, What kind of a girl does science? The construction of school science identities, J. Res. Sci. Teach. 37, 441 (2000).

[7] J. Lave and E. Wenger, Situated Learning: Legitimate Peripheral Participation (Cambridge University Press, Cambridge, England, 1991).

[8] E. Wenger, Communities of practice and social learning systems, Organization 7, 225 (2000).

[9] A. Amin and J. Roberts, Knowing in action: Beyond communities of practice, Res. Policy 37, 353 (2008).

[10] J. Lave, Situated learning and changing practice, in Community, Economic Creativity, and Organisation (Oxford University Press, Oxford, 2008), pp. 283-296.

[11] J. Lemke, Cognition, context and learning: A social semiotic perspective, in Situated Cognition: Social, Semiotic, and Psychologival Perspectives (Erlbaum, Mahwah, NJ, 1997), pp. 37-56.

[12] M. Boylan, Deepening system leadership: Teachers leading from below, Educ. Manage. Adm. Leadership 44, 57 (2010).

[13] P. Irving, M. J. Obsniuk, and M. D. Caballero, P3: A practice focused learning environment, Eur. J. Phys. 38, 055701 (2017).

[14] R. Ivie, G. Anderson, and S. White, Statistical Research Center of the American Institute of Physics (A product of the Statistical Research Center of the American Institute of Physics 1 Physics Ellipse, College Park, MD, 2014).

[15] https://www.onlineuniversities.com/blog/2012/06/10startling-stats-about-minorities-stem/ (2012).

[16] https://physicsworld.com/a/gender-gap-in-physicsamongst-highest-in-science/ (2018).

[17] National Science Foundation, Women, Minorities, and Persons with Disabilities in Science and Engineering (National Center for Science and Engineering Statistics Directorate for Social, Behavioral and Economic Sciences, Washington, DC, 2017), https://www.nsf.gov/statistics/ 2017/nsf17310/static/downloads/nsf17310-digest.pdf (2017).

[18] P. J. Mulvey and S. Nicholson, Physics Bachelors Degrees (AIP Research Center, College Park, MD, 2012).

[19] P.W. Irving and E. C. Sayre, Becoming a physicist: The roles of research, mindsets, and milestones in upper-division student perceptions, Phys. Rev. ST Phys. Educ. Res. 11, 020120 (2015).

[20] D. Dedra and S. Li, Promoting productive communities of practice: an instructor's perspective, AIP Conf. Proc. 1179, 125 (2009).

[21] O. Pierrakos, T. K. Beam, J. Constantz, A. Johri, and R. Anderson, On the development of a professional identity: Engineering persisters vs engineering switchers, in Proceedings of the 2009 39th IEEE Frontiers in Education Conference (IEEE, Bellingham, WA, 2009), pp. 1-6.

[22] C. M. Johnson, A survey of current research on online communities of practice, Internet Higher Educ. 4, 45 (2001).

[23] R. Oliver and J. Herrington, Using situated learning as a design strategy for Web-based learning, in Instructional and Cognitive Impacts of Web-Based Education (IGI Global, Hershey, PA, 2000), pp. 178-191.

[24] S. A. Barab and T. Duffy, From practice fields to communities of practice, in Theoretical Foundations of Learning Environments, edited by D. H. Jonassen and S. M. Land (Lawrence Erlbaum Associates, London, 2000), pp. 25-55, https://citeseerx.ist.psu.edu/viewdoc/ download?doi=10.1.1.587.541\&rep=rep1\&type=pdf.

[25] M. Laukenmann, M. Bleicher, S. Fuss, M. Glaser-Zikuda, P. Mayring, and C. von Rhöneck, An investigation of the influence of emotional factors on learning in physics instruction, Int. J. Sci. Educ. 25, 489 (2003).

[26] S. Vahedi and M. Yari, Role of cognitive and emotional factors on educational achievement among high school students in physics, Eur. Online J. Nat. Soc. Sci. 3, 572 (2014), http://european-science.com/eojnss/article/view/ 891.

[27] J. Whitcomb, H. Borko, and D. Liston, Growing Talent: Promising Professional Development Models and Practices (Sage Publications, Los Angeles, CA, 2009).

[28] D. Bennett and S. A. Male, A student-staff community of practice within an inter-university final-year project, in Implementing Communities of Practice in Higher Education (Springer, New York, 2017), pp. 325-346.

[29] T. Cochrane and V. Narayan, Principles of modeling COPs for pedagogical change: Lessons learnt from practice 2006 to 2014, in Implementing Communities of Practice in Higher Education (Springer, New York, 2017), pp. 619-643.

[30] K. Patton and M. Parker, Teacher education communities of practice: More than a culture of collaboration, Teaching Teacher Educ. 67, 351 (2017).

[31] C. Giles and A. Hargreaves, The sustainability of innovative schools as learning organizations and professional learning communities during standardized reform, Educ. Admin. Quart. 42, 124 (2006).

[32] N. S. Board, Next Generation Science Standards: For States, by States (The National Academies Press, Washington, DC, 2013).

[33] S. A. Weatherford, Student use of Physics to Make Sense of Incomplete but Functional VPython Programs in a Lab Setting (North Carolina State University, Raleigh, NC, 2011). 
[34] J. Lave, Teaching, as learning, in practice, Mind Culture Activity 3, 149 (1996).

[35] L. R. Van Zoest and J. Bohl, Mathematics teacher identity: a framework for understanding secondary school mathematics teachers' learning through practice, Teach. Develop. 9, 315 (2005), https://www.tandfonline.com/ doi/abs/10.1080/13664530500200258.

[36] E. Wenger, Conceptual tools for COPs as social learning systems: Boundaries, identity, trajectories and participation, in Social Learning Systems and Communities of Practice (Springer, New York, 2010), pp. 125-143.

[37] D. M. Gilbuena, B. U. Sherrett, E. S. Gummer, A. B. Champagne, and M. D. Koretsky, Feedback on professional skills as enculturation into communities of practice, J. Eng. Educ. 104, 7 (2015).

[38] P. W. Irving and E. C. Sayre, Identity statuses in upperdivision physics students, Cultural Studies Sci. Educ. 11, 1155 (2016).

[39] J. Morton, Communities of practice in higher education: A challenge from the discipline of architecture, Linguist. Educ. 23, 100 (2012).

[40] E. C. Wenger and W. M. Snyder, Communities of practice: The organizational frontier, Harv. Bus. Rev. 78, 139 (2000), https://hbr.org/2000/01/communities-of-practicethe-organizational-frontier.

[41] J. Boaler, Mathematics from another world: Traditional communities and the alienation of learners, J. Math. Behav. 18, 379 (2000).

[42] E. Nardi and S. Steward, Is mathematics T.I.R.E.D? A profile of quiet disaffection in the secondary mathematics classroom, Br. Educ. Res. J. 29, 345 (2003).

[43] P.-L. Hsu and W.-M. Roth, From a sense of stereotypically foreign to belonging in a science community: Ways of experiential descriptions about high school students' science internship, Res. Sci. Educ. 40, 291 (2010).

[44] P. R. Aschbacher, E. Li, and E. J. Roth, Is science me? High school students' identities, participation and aspirations in science, engineering, and medicine, J. Res. Sci. Teach. 47, 564 (2010).

[45] G. S. Aikenhead, Science education: Border crossing into the subculture of science, Stud. Sci. Edu. 27, 1 (1996).

[46] Z. Hazari, G. Sonnert, P. M. Sadler, and M.-C. Shanahan, Connecting high school physics experiences, outcome expectations, physics identity, and physics career choice: A gender study, J. Res. Sci. Teach. 47, 978 (2010).

[47] G. Potvin and Z. Hazari, The development and measurement of identity across the physical sciences, in Proceedings of the 2013 Physics Education Research Conference, Portland, OR (AIP, New York, 2013).

[48] S. L. Li and M. E. Loverude, Identity and belonging: Are you a physicist (chemist)?, AIP Conf. Proc. 1513, 246 (2013).

[49] K. Funkhouser, M. D. Caballero, P. V. Irving, and V. Sawtelle, What counts in laboratories: toward a practicebased identity survey, arXiv:1807.03717.

[50] P. W. Irving and E. C. Sayre, Conditions for building a community of practice in an advanced physics laboratory, Phys. Rev. ST Phys. Educ. Res. 10, 010109 (2014).
[51] P. Irving and E. Sayre, Physics identity development: A snapshot of the stages of development of upper-level physics students, J. Scholarship Teach. Learn. 13, 68 (2013), https://scholarworks.iu.edu/journals/index.php/ josotl/article/view/3521.

[52] K. Handley, A. Sturdy, R. Fincham, and T. Clark, Within and beyond communities of practice: Making sense of learning through participation, identity and practice, J. Manage. Studies 43, 641 (2006).

[53] A. Sfard, On two metaphors for learning and the dangers of choosing just one, Educ. Res. 27, 4 (1998).

[54] V. L. Akerson, T. A. Cullen, and D. L. Hanson, Fostering a community of practice through a professional development program to improve elementary teachers' views of nature of science and teaching practice, J. Res. Sci. Teach. 46, 1090 (2009).

[55] E. Wegner and M. Nuckles, Knowledge acquisition or participation in communities of practice? Academics' metaphors of teaching and learning at the university, Studies Higher Educ. 40, 624 (2015).

[56] E. Wenger, R. A. McDermott, and W. Snyder, Cultivating Communities of Practice: A Guide to Managing Knowledge (Harvard Business Press, Cambridge, MA, 2002).

[57] M. Denscombe, Communities of practice: A research paradigm for the mixed methods approach, J. Mixed Methods Res. 2, 270 (2008).

[58] L. M. Avery and W. S. Carlsen, Knowledge, identity, and teachers' multiple communities of practice, in Proceedings of the National Association for Research in Science Teaching, St. Louis, MO (Cornell University, Ithaca, 2001).

[59] A. S. Palincsar, S. J. Magnusson, N. Marano, D. Ford, and N. Brown, Designing a community of practice: Principles and practices of the GIsML community, Teaching Teacher Educ. 14, 5 (1998).

[60] S. Gherardi, D. Nicolini, and F. Odella, Toward a social understanding of how people learn in organizations: The notion of situated curriculum, Manage. Learn. 29, 273 (1998).

[61] L. Parker and D. Galloway, Creative communities: Shaping process through performance and play, Trans. Digital Games Res. Assoc. 3, 57 (2017), https://kilthub .cmu.edu/articles/Transactions_of_the_Digital_Games_ Research_Association_--_Vol_3_No_2/6687002.

[62] I. Pyrko, V. Dorfler, and C. Eden, Thinking together: What makes Communities of Practice work?, Hum. Relations 70, 389 (2017).

[63] H. Saint-Onge and D. Wallace, Leveraging Communities of Practice for Strategic Advantage (Routledge, London, 2012).

[64] D. M. Culver and P. Trudel, Cultivating coaches' communities of practice: Developing the potential for learning through interactions, in The Sports Coach as Educator (Routledge, London, 2006), pp. 115-130.

[65] https://wenger-trayner.com/introduction-to-communitiesof-practice/ (2019).

[66] C. Power and L. Armstrong, A facilitated community of practice: Enabling student success in the blended learning environment, in Implementing Communities 
of Practice in Higher Education (Springer, New York, 2017), pp. 227-252.

[67] P. Irving and M. Caballero, Understanding the PICUP community of practice, in Proceedings of the Physics Education Research Conference, Cincinnati, OH (AIP, New York, 2017), pp. 188-191.

[68] A. Ardichvili, V. Page, and T. Wentling, Motivation and barriers to participation in virtual knowledge-sharing communities of practice, J. Knowl. Manage. 7, 64 (2003).

[69] L. Gauthier, Redesigning for student success: Cultivating communities of practice in a higher education classroom, J. Scholarship Teach. Learn. 16, 1 (2016).

[70] A. Cox, What are communities of practice? A comparative review of four seminal works, J. Info. Sci. 31, 527 (2005).

[71] E. C. Cheng, Cultivating communities of practice via learning study for enhancing teacher learning, in Global Learn (Association for the Advancement of Computing in Education, Waynesville, NC, 2011), pp. 1138-1152.

[72] L. Houghton, A. Ruutz, W. Green, and R. Hibbins, I just do not have time for new ideas: resistance, resonance and micro-mobilisation in a teaching community of practice, Higher Educ. Res. Dev. 34, 527 (2015).

[73] J. Preece, Etiquette, empathy and trust in communities of practice: Stepping-stones to social capital, J. Univers. Comput. Sci. 10, 294 (2004), http://www.jucs.org/ jucs_10_3/etiquette_empathy_and_trust/Preece_J.pdf.

[74] K. Wathne, J. Roos, and G. Von Krogh, Managing Knowledge-Perspectives on Cooperation and Competition (Sage Publications, 1996), pp. 55-81.

[75] M. Schultz and G. O'Brien, The Australian chemistry discipline network: A supportive community of practice in a hard science, in Implementing Communities of Practice in Higher Education (Springer, New York, 2017), pp. 501-530.

[76] A. L. deChambeau, Supported student success: Communities of practice in higher education, Ph.D. thesis, Prescott College, 2014.

[77] T. Murphey, J. Falout, Y. Fukada, and T. Fukuda, Group dynamics: Collaborative agency in present communities of imagination, in Psychology for Language Learning (Springer, New York, 2012).

[78] A. Fuller, H. Hodkinson, P. Hodkinson, and L. Unwin, Learning as peripheral participation in communities of practice: a reassessment of key concepts in workplace learning, Br. Educ. Res. J. 31, 49 (2005).

[79] B. Baran and K. Cagiltay, Motivators and Barriers in the Development of Online Communities of Practice. Eurasian Journal of Educational Research (EJER), pp. 79-96, https://www.researchgate.net/publication/267981588_ Motivators_and_Barriers_in_the_Development_o f_ Online_Communities_of_Practice_Suggested_Citation.

[80] J. Collier and R. Esteban, Governance in the participative organisation: Freedom, creativity and ethics, J. Business Ethics 21, 173 (1999).

[81] D. Moulton, P. Corcoran, P. James, and P. Rathore, A community of practice for blended science and engineering learning and teaching at UniSA, in Implementing Communities of Practice in Higher Education (Springer, New York, 2017), pp. 253-280.
[82] W. Barber, S. King, and S. Buchanan, Problem based learning and authentic assessment in digital pedagogy: Embracing the role of collaborative communities, Electronic J. E-Learn. 13, 59 (2015), https://eric.ed.gov/? id=EJ1060176.

[83] M. Goos, P. Galbraith, and P. Renshaw, Establishing a community of practice in a secondary mathematics classroom, in Mathematics Education (Routledge, London, 2003), pp. 101-126.

[84] R. Brown, Exploring the social positions that students construct within a classroom community of practice, Int. J. Educ. Res. 46, 116 (2007).

[85] N. Kapucu, Classrooms as communities of practice: Designing and facilitating learning in a networked environment, J. Public Affairs Educ. 18, 585 (2012).

[86] E. Tan and A. C. Barton, From peripheral to central, the story of Melanie's metamorphosis in an urban middle school science class, Sci. Educ. 92, 567 (2008).

[87] N. Pratt and J. Back, Spaces to discuss mathematics: communities of practice on an online discussion board, Res. Math. Educ. 11, 115 (2009).

[88] E. Yackel and P. Cobb, Sociomathematical norms, argumentation, and autonomy in mathematics, J. Res. Math. Educ. 4, 458 (1996).

[89] Engineering Practice In A Global Context, edited by B. Williams, J. Figueiredo, and J. Trevelyan (CRC Press, London, 2013), https://www.taylorfrancis.com/books/e/ 9780429227851.

[90] E. C. Sayre, C. Fracchiolla, and B. Van Dusen, iPad screencasts as evidence of accountable disciplinary knowledge, arXiv:1605.08105.

[91] R. Butler, Task-involving and ego-involving properties of evaluation: Effects of different feedback conditions on motivational perceptions, interest, and performance, J. Educ. Psychol. 79, 474 (1987).

[92] R. Butler, Enhancing and undermining intrinsic motivation: The effects of task-involving and ego-involving evaluation on interest and performance, Br. J. Educ. Psychol. 58, 1 (1988).

[93] C.S. Dweck, Self-theories: Their role in Motivation, Personality and Development (Psychology Press, Philadelphia, PA, 2000).

[94] J. Dolin, P. Black, W. Harlen, and A. Tiberghien, Exploring relations between formative and summative assessment, in Transforming Assessment (Springer, New York, 2018), pp. 53-80.

[95] F. Marton and R. Saljo, Approaches to Learning (Scottish Academic Press, Edinburgh, 1997).

[96] P. Irving, A phenomenographic study of introductory physics students: Approaches to their learning and perceptions of their learning environment in a physics problem-based learning environment, Ph.D. thesis, Dublin Institute of Technology, 2010.

[97] A. A. Callender, A. M. Franco-Watkins, and A. S. Roberts, Improving metacognition in the classroom through instruction, training, and feedback, Metacognition Learn. 11, 215 (2016).

[98] S. J. Thompson, M. L. Thurlow, and D. Malouf, Creating better tests for everyone through universally 
designed assessments, J. Appl. Testing Technol. 10 (2004), http://www.jattjournal.com/index.php/atp/article/ view/48341.

[99] P. C. Hamerski, P. Irving, and D. McPadden, Learning assistants as constructors of feedback: How are they impacted?, in Proceedings of the 2018 Physics Education Research Conference, Washington, DC (AIP, New York, 2018).

[100] P. Irving, V. Sawtelle, and M. Caballero, Formative feedback in $\mathrm{P}^{3}$ (Projects and Practices in Physics), in Proceedings of the 2015 Physics Education Research Conference, College Park, MD (AIP, New York, 2015).

[101] K. Griswold, D. McPadden, M. D. Caballero, and P. Irving, Denoting and comparing leadership attributes and behaviors in group work, in Proceedings of the 2018 Physics Education Research Conference, Washington, DC (AIP, New York, 2018).

[102] D. McPadden, P. C. Hamerski, M. D. Caballero, and P. W. Irving, Feedback as a mechanism for improving students' scientific communication skills, in Proceedings of the 2018 Physics Education Research Conference, Washington, DC (AIP, New York, 2018).

[103] J. B. Biggs, From theory to practice: A cognitive systems approach, Higher Educ. Res. Dev. 12, 73 (1993).

[104] J.B. Biggs, What the student does: Teaching for enhanced learning, Higher Educ. Res. Dev. 18, 57 (1999).

[105] A. K. Bednar, D. Cunningham, T.M. Duffy, and J. D. Perry, Theory into practice: How do we link?, in Instructional technology: Past, present, and future, edited by G. J. Anglin (Libraries Unlimited, Englewood, CO, 1992).

[106] D. H. Jonassen, Instructional design models for well-structured and III-structured problem-solving learning outcomes, Educ. Technol. Res. Dev. 45, 65 (1997).

[107] B. K. Hofer and P. R. Pintrich, The development of epistemological theories: Beliefs about knowledge and knowing and their relation to learning, Rev. Educ. Res. 67, 88 (1997).

[108] C. Chin and L.-G. Chia, Problem-based learning: Using ill-structured problems in biology project work, Sci. Educ. 90, 44 (2006).

[109] K. M. Edens, Preparing problem solvers for the 21st century through problem-based learning, Coll. Teach. 48, 55 (2000).

[110] L. Torp and S. Sage, Problems as Possibilities: ProblemBased Learning for K-12 Education (Association for Supervision and Curriculum Development, Alexandria, Virginia, 1998).

[111] H. S. Barrows and R.M. Tamblyn, Problem-Based Learning: An Approach to Medical Education (Springer, New York, 1980).

[112] S. M. Brookhart, How to Assess Higher-Order Thinking Skills in your Classroom (ASCD, Alexandria, Virginia USA, 2010).

[113] J. Bergmann and A. Sams, Flip your Classroom: Reach every Student in Every Class Every Day (International
Society for Technology in Education, Washington DC, 2012).

[114] W.-M. Roth, Where is the context in contextual word problem?: Mathematical practices and products in grade 8 students' answers to story problems, Cognit. Instr. 14, 487 (1996).

[115] J. Savery and T. Duffy, Problem based learning: An instructional model and its constructivist framework, Educ. Technol. 35, 31 (1995), https://www.jstor.org/ stable/44428296?seq $=1$.

[116] C. Bereiter, Implications of postmodernism for science, or, science as progressive discourse, Educ. Psychol. 29, 3 (1994).

[117] M. Scardamalia and C. Bereiter, Computer support for knowledge-building communities, J. Learn. Sci. 3, 265 (1994).

[118] H. S. Barrows and A. Myers, Unpublished monograph (Problem-Based Learning Institute, Lanphier High School and Southern Illinois University Medical School, Springfield, IL, 1993).

[119] K. Bielaczyc and A. Collins, Learning communities in classrooms: A reconceptualization of educational practices, in Instructional Design Theories and Models: A New Paradigm of Instructional Theory (Lawrence Erlbaum, New Jersey, 1999), pp. 269-292.

[120] R. M. Palloff and K. Pratt, Building Learning Communities in Cyberspace (Jossey-Bass, San Francisco, 1999), Vol. 12.

[121] J. Rogers, Communities of practice: A framework for fostering coherence in virtual learning communities, Educ. Technol. Soc. 3, 384 (2000), https://www.jstor .org/stable/jeductechsoci.3.3.384?seq=1\#metadata_info_ tab_contents.

[122] K. D. Squire and C. B. Johnson, Supporting distributed communities of practice with interactive television, Educ. Technol. Res. Dev. 48, 23 (2000).

[123] S. M. Powers and S. Guan, Examining the range of student needs in the design and development of a webbased course, in Instructional and Cognitive Impacts of Web-Based Education (IGI Global, Hershey, PA, 2000), pp. 200-216.

[124] A. Schoenfeld, In fostering communities of inquiry, must it matter that the teacher knows the "answer"?, Learn. Math. 26, 11 (1996), https://flm-journal.org/Articles/ 5226E38BEF5A8F9AA1A96FB4866A55.pdf.

[125] D. Kaufman and K. V. Mann, Understanding medical education: Evidence, Theory and Practice (WileyBlackwell, West Sussex, UK, 2014), pp. 7-30.

[126] C. Hoadley, What is a Community of Practice and how can we support it?, in Theoretical Foundations of Learning Environments, 2nd ed., edited by D. H. Jonassen and S. M. Land (Routledge, New York, 2012), pp. 287-300.

[127] J. Lave, The culture of acquisition and the practice of understanding, in Cultural Psychology: Essays on Comparative Human Development, edited by J. Stigler, R. Schweder, and G. Herdt (Cambridge University Press, Cambridge, England, 1997), pp. 309-327, https://doi.org/ 10.1017/CBO9781139173728.010. 
[128] J. Roberts, Limits to communities of practice, J. Manage. Stud. 43, 623 (2006).

[129] A. Pawlak, Collaborative learning environments in introductory physics, Ph.D. thesis, Michigan State University, East Lansing, MI, 2018.

[130] K. L. Tonso, Student engineers and engineer identity: Campus engineer identities as figured world, Cultural Studies Sci. Edu. 1, 273 (2006).
[131] E. Seymour, The loss of women from science, mathematics, and engineering undergraduate majors: An explanatory account, Sci. Educ. 79, 437 (1995).

[132] K. Topping, Self and peer assessment in school and university: Reliability, validity and utility, in Optimising New Modes of Assessment: In Search of Qualities and Standards (Springer, Dordrecht, 2003). 